\title{
RESEARCH
}

Open Access

\section{Prediction of differentially expressed microRNAs in blood as potential biomarkers for Alzheimer's disease by meta-analysis and adaptive boosting ensemble learning}

Sze Chung Yuen ${ }^{1 \dagger}$, Xiaonan Liang ${ }^{1 \dagger}$, Hongmei Zhu' ${ }^{1}$, Yongliang Jia ${ }^{1,2,3}$ and Siu-wai Leung ${ }^{4,5^{*}}$

\begin{abstract}
Background: Blood circulating microRNAs that are specific for Alzheimer's disease (AD) can be identified from differentially expressed microRNAs (DEmiRNAs). However, non-reproducible and inconsistent reports of DEmiRNAs hinder biomarker development. The most reliable DEmiRNAs can be identified by meta-analysis. To enrich the pool of DEmiRNAs for potential AD biomarkers, we used a machine learning method called adaptive boosting for miRNA disease association (ABMDA) to identify eligible candidates that share similar characteristics with the DEmiRNAs identified from meta-analysis. This study aimed to identify blood circulating DEmiRNAs as potential AD biomarkers by augmenting meta-analysis with the ABMDA ensemble learning method.

Methods: Studies on DEmiRNAs and their dysregulation states were corroborated with one another by metaanalysis based on a random-effects model. DEmiRNAs identified by meta-analysis were collected as positive examples of miRNA-AD pairs for ABMDA ensemble learning. ABMDA identified similar DEmiRNAs according to a set of predefined criteria. The biological significance of all resulting DEmiRNAs was determined by their target genes according to pathway enrichment analyses. The target genes common to both meta-analysis- and ABMDAidentified DEmiRNAs were collected to construct a network to investigate their biological functions.
\end{abstract}

\footnotetext{
* Correspondence: siuwai.leung@ed.ac.uk

†'Sze Chung Yuen and Xiaonan Liang are co-first authors

${ }^{4}$ Shenzhen Institute of Artificial Intelligence and Robotics for Society, Shenzhen, China

${ }^{5}$ Edinburgh Bayes Centre for Al Research in Shenzhen, College of Science and Engineering, University of Edinburgh, Edinburgh, Scotland, UK Full list of author information is available at the end of the article
}

(c) The Author(s). 2021 Open Access This article is licensed under a Creative Commons Attribution 4.0 International License, which permits use, sharing, adaptation, distribution and reproduction in any medium or format, as long as you give appropriate credit to the original author(s) and the source, provide a link to the Creative Commons licence, and indicate if changes were made. The images or other third party material in this article are included in the article's Creative Commons licence, unless indicated otherwise in a credit line to the material. If material is not included in the article's Creative Commons licence and your intended use is not permitted by statutory regulation or exceeds the permitted use, you will need to obtain permission directly from the copyright holder. To view a copy of this licence, visit http://creativecommons.org/licenses/by/4.0/. The Creative Commons Public Domain Dedication waiver (http://creativecommons.org/publicdomain/zero/1.0/) applies to the data made available in this article, unless otherwise stated in a credit line to the data. 
Results: A systematic database search found 7841 studies for an extensive meta-analysis, covering 54 independent comparisons of 47 differential miRNA expression studies, and identified 18 reliable DEmiRNAs. ABMDA ensemble learning was conducted based on the meta-analysis results and the Human MicroRNA Disease Database, which identified 10 additional AD-related DEmiRNAs. These 28 DEmiRNAs and their dysregulated pathways were related to neuroinflammation. The dysregulated pathway related to neuronal cell cycle re-entry (CCR) was the only statistically significant pathway of the ABMDA-identified DEmiRNAs. In the biological network constructed from 1865 common target genes of the identified DEmiRNAs, the multiple core ubiquitin-proteasome system, that is involved in neuroinflammation and CCR, was highly connected.

Conclusion: This study identified 28 DEmiRNAs as potential AD biomarkers in blood, by meta-analysis and ABMDA ensemble learning in tandem. The DEmiRNAs identified by meta-analysis and ABMDA were significantly related to neuroinflammation, and the ABMDA-identified DEmiRNAs were related to neuronal CCR.

Keywords: Alzheimer's disease, Meta-analysis, MicroRNAs, ABMDA, Biomarkers, Neuroinflammation, Neuronal cell cycle re-entry

\section{Background}

Alzheimer's disease (AD) is subcellularly characterized by the presence of extracellular amyloid-beta $(A \beta)$ plaque deposition and intracellular neurofibrillary tangles of hyperphosphorylated tau proteins [1]. The aberrant protein aggregates are accompanied by activation of neuroinflammation, and loss of synaptic functions [2]. In the progression of $\mathrm{AD}$, irreversible loss of neurons and synaptic functions gradually develops over decades before the manifestation of cognitive symptoms [3]. Because the root causes of pathological $A \beta$ accumulation and hyperphosphorylated tau proteins are not clear, drug development for $\mathrm{AD}$ often fails and current $\mathrm{AD}$ treatments alleviate symptoms only. The failure of most clinical trials in $\mathrm{AD}$ has been partially attributed to the lack of sensitive biomarkers to identify potential AD [4], which can identify and enroll patients at the early stage of $\mathrm{AD}$, as it may be too late to rescue the dysfunction present in advanced stages of the disease.

The National Institute of Neurological and Communicative Disorders and Stroke and the Alzheimer's Disease and Related Disorders Association (NINCDS-ADRDA) and the National Institute on Aging and Alzheimer's Association (NIA-AA) proposed that the diagnosis of $\mathrm{AD}$ should be dependent on biomarkers rather than solely dependent on clinical symptoms $[5,6]$. Aberrant levels of $A \beta$ and tau proteins in cerebrospinal fluid (CSF) and blood have been evaluated as biomarkers for AD diagnosis $[7,8]$, specifically, increased levels of total and hyperphosphorylated tau proteins, and decreased levels of $A \beta$ in CSF. CSF levels of total and hyperphosphorylated tau proteins are correlated with neurofibrillary tangle load, and CSF levels of $A \beta$ are inversely correlated with amyloid load [9]. However, a review concluded that these CSF biomarkers are more useful for ruling out AD, than for indicating a definite diagnosis [10]. To avoid the side effects of invasive CSF sampling, such as positional headache, Fei et al. [11] have proposed the use of peripheral blood $A \beta$ detection, such as the ratio of $A \beta 42 /$ $\mathrm{A} \beta 40$. However, another review has reported that lower plasma $A \beta 42 / A \beta 40$ ratios might not be associated with increased AD risk [12]. The plasma phosphorylated tau 217 is proposed to distinguish AD from non-AD neurodegenerative individuals, since its level increases more steeply in non-demented individuals with amyloid positivity than those without amyloid positivity [13]. The limitations to use $A \beta$ or tau proteins as biomarkers in blood might be due to several reasons. First, $A \beta$ in both CSF and blood tends to self-aggregate [14], masking epitopes for detection and reducing the correlation with $A D$. Second, the entrance of $A \beta$ and tau proteins into body fluid is hindered by the extent of blood-brain barrier (BBB) leakage [15]. Thus, there is a need to identify other potential non-invasive biomarkers to aid the diagnosis of AD. An ideal AD biomarker would allow for mass screening to identify patients at high risk of developing $\mathrm{AD}$ in the presymptomatic stage with adequate reliability.

MicroRNAs (miRNAs) are a class of single-stranded non-coding RNAs that are 18-25 nucleotides in length and bind to the 3' untranslated region of target mRNAs to modify the target mRNAs' expression in a posttranscriptional manner [16]. Each miRNA simultaneously targets hundreds of mRNAs, and over 2500 mature miRNAs have been recorded in the latest version of the miRBase database [17]. Out of all recorded miRNAs, 300 have been associated with neurodegenerative diseases and 131 miRNAs are specific for AD [18]. Downregulated expressions of $\mathrm{miR}-29 \mathrm{a} / \mathrm{b}-1, \mathrm{miR}-29 \mathrm{c}$, and miR-339-5p have been reported to upregulate the expression of $B A C E 1$ in $A D$ brain, thereby increasing $A \beta$ production [19-21]. The expression of miR-15/107 in 
cerebral cortical gray matter is correlated with amyloid plaque density [22]. The AD-dysregulated miRNAs in brain have been associated with neuroinflammation and cell cycle regulation [23, 24] and may be released into peripheral blood through the BBB [25] and transported by lipoproteins in circulation for stability [26]. These stable AD-dysregulated miRNAs in blood may reflect the composition of dysregulated miRNAs in brain, suggesting that they may be potential biomarkers of $\mathrm{AD}$. The dysregulated miRNAs were found in plasma and serum of $A D$ patients $[27,28]$, e.g., the expression of miR-125b in serum of AD patients is correlated with Mini-Mental State Examination (MMSE) scores [29]. Compared with biomarkers in brain or CSF, blood circulating biomarkers are preferable for their higher accessibility. However, before establishing any miRNAs as AD biomarkers, it is necessary to first evaluate their reliability and consistency among different studies of differentially expressed miRNAs (DEmiRNAs) in AD patients, and their biological significance to understand their roles in the pathogenesis of AD.

Thus far, the development of blood circulating DEmiRNAs as AD biomarkers has been hindered by inconsistent and unreliable studies. For example, Denk et al. [30] and Wu et al. [31] conducted similar studies using quantitative real-time polymerase chain reaction (qRT-PCR) to quantify the expression of miRNAs in serum, and found 22 and 9 DEmiRNAs, respectively. Only one DEmiRNA, miR-146a-5p, was common between the two studies. Further, the studies reported the same miR-146a-5p to have opposite directions of dysregulation in AD. This inconsistency can be attributed to differences in the AD patients recruited for the trials, including the presence of other disease conditions that might influence the levels of biological molecules in blood, and the stages of disease progression [32, 33]. A meta-analysis of these inconsistent DEmiRNA results may resolve discrepancies and enhance generalizability of the results. Takousis et al. [34] conducted a metaanalysis to identify DEmiRNAs in brain, blood, and CSF from expression profiling studies of AD. They applied Stouffer's method to integrate the $P$ values of every DEmiRNA from each independent study and found 32 statistically significant DEmiRNAs in blood. However, Stouffer's method does not include information about dysregulation states of DEmiRNAs and its use of $P$ values is outdated in terms of the methodology of metaanalysis; thus, the results were probably biased by the number of studies reporting the same DEmiRNAs. Hu et al. and Zhang et al. [35, 36] also conducted metaanalyses independently and reported that the sensitivity ( $86 \%$ and $80 \%$, respectively) and specificity ( $87 \%$ and $83 \%$, respectively) of blood-based miRNAs are comparable with those of fluorodeoxyglucose-positron emission tomography (FDG-PET; sensitivity: 91\%, specificity: 86\%) [37] for AD diagnosis. The results of these metaanalyses demonstrate that some blood circulating DEmiRNAs may be useful for AD diagnosis, but there has not been extensive identification of DEmiRNAs that may serve as $\mathrm{AD}$ biomarkers.

Structural and functional patterns of similar DEmiRNAs can be identified by machine learning, which has been applied to the identification of $\mathrm{AD}$ biomarkers. Studies using different machine learning techniques have identified different DEmiRNAs for $\mathrm{AD}$ diagnosis with up to $89 \%$ accuracy [38-40]. Until recently, machine learning mainly focused on fine tuning the set of DEmiRNAs identified from differential miRNA expression profiling studies to obtain a smaller panel of miRNAs for AD diagnosis. As an emerging trend, machine learning is being used to predict potential DEmiRNAs as AD biomarkers based on DEmiRNAs identified from differential expression profiling studies. For example, Zhao et al. [41] used the adaptive boosting for miRNA disease association (ABMDA) ensemble learning method to identify miRNAs that are associated with a disease. ABMDA is a supervised learning approach with validation against the Human MicroRNA Disease Database (HMDD) [42]. Its clustering algorithm is based on $k$ means distance, and its boosting technique combines classifiers by their corresponding weights to form a stronger classifier. The ABMDA ensemble learning method relies upon the initial set of DEmiRNAs to predict additional DEmiRNAs. In the present study, we used meta-analysis to enhance the reliability of the initial DEmiRNAs and augment the prediction performance of the ABMDA ensemble learning method.

This study aimed to identify blood circulating DEmiRNAs as potential AD biomarkers by augmenting the DEmiRNAs identified by meta-analysis with the ABMDA ensemble learning method. The meta-analysis was conducted under the guidance of the PRISMA statement [43], with the adjustment in the risk of bias assessment.

\section{Methods}

The present meta-analysis was mainly conducted using $\mathrm{R}$ and Python packages and is illustrated in Fig. 1. If an included study contained several comparisons, the DEmiRNAs from each comparison were collected independently. The sample sizes of control and AD groups, and DEmiRNAs and their dysregulated states from each independent comparison were extracted. The names of all reported DEmiRNAs were standardized based on the miRBase database [17] before the meta-analysis. A metaanalysis for the DEmiRNAs was performed when more than one comparison reported the same DEmiRNA. Statistically significant DEmiRNAs from the meta-analysis 


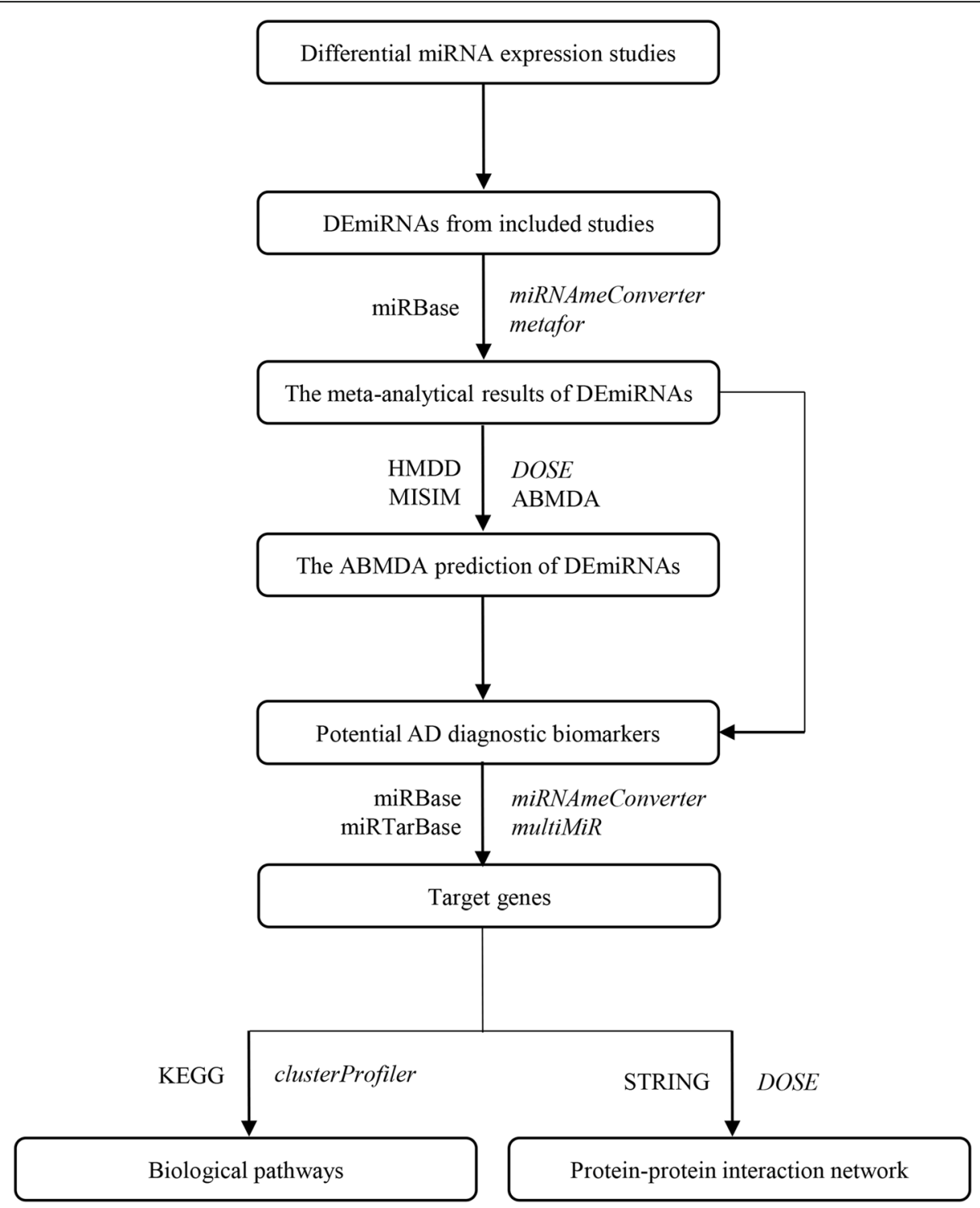

Fig. 1 Overall study design. The italic font and normal font on the right-hand side of the arrows represent the R package and python-based algorithm, respectively. The name of each database is shown on the left-hand side of the arrow

were collected as positive miRNA-AD associations for the ABMDA ensemble learning method to further increase the number of positive miRNA-AD associations. The ABMDA-identified miRNAs with scores higher than the predefined criteria, together with the DEmiRNAs identified by the meta-analysis, were treated as potential AD biomarkers in blood. The biological significance of the identified DEmiRNAs was studied by a biological pathway enrichment analysis and network analysis of their corresponding target genes. The combination of meta-analysis and ABMDA ensemble learning might be beneficial to resolve the existing inconsistencies and perform systematical predictions based on reliable results.

\section{Differential miRNA expression studies selection}

Differential miRNA expression studies were collected from PubMed from inception until January 10, 2020, from ScienceDirect and Web of Science between 1993 and 2019. The search strategy is shown below:

For PubMed, ("Alzheimer's disease" [MESH Terms] OR "Alzheimer's disease" [All Fields] OR "alzheimer" [MESH Terms] OR "alzheimer" [All Fields]) AND ("microRNA" [MESH Terms] OR "microRNA" [All Fields] OR "miRNA" [MESH Terms] OR "miRNA*" [All Fields])

For ScienceDirect and Web of Science, (microRNA OR miRNA) AND Alzheimer

After the search, two authors (SCY and XNL) screened all titles, abstracts, and full texts independently 
according to the eligibility criteria. Disagreements between the authors were resolved by discussion with the other authors.

The case-control studies reporting differential miRNA expression in blood from $\mathrm{AD}$ and healthy participants were included. The included studies had to report the differential expression profiling methods, sample sizes of the disease and control groups, and statistical significance for each DEmiRNA. Studies were excluded if they (1) were not $\mathrm{AD}$ research (i.e., focused on other dementia disease conditions); (2) reported irrelevant effects on differential miRNA expression profiles (i.e., demonstrated treatment effects on the differential miRNA expression); (3) did not report the blood DEmiRNAs for $\mathrm{AD}$ and healthy participants (i.e., were not controlled studies, or recruited AD participants suffering from other diseases); (4) did not report DEmiRNAs and their corresponding dysregulation states (i.e., up or down) as outcomes; or (5) did not obtain DEmiRNAs by a validation method (i.e., qRT-PCR).

\section{Meta-analysis}

For each study, the following data were extracted for the meta-analysis: (1) comparison ID (PubMed ID with blood elements or disease stages); (2) blood elements; (3) differential miRNA expression profiling method; (4) number of AD cases; (5) number of control cases; and (6) DEmiRNAs and their dysregulated states. For studies that reported both screening and validation results of differential miRNA expression, only the results from validation methods were collected. The names of extracted DEmiRNAs were standardized using the miRNAmeConverter [44] package for $\mathrm{R}$ software with the miRBase database [17]. For each DEmiRNA that was reported by more than one comparison, a meta-analysis was performed using the metafor [45] package for $\mathrm{R}$ software. The meta-analysis was conducted for each qualified DEmiRNA, independently, under a random-effects model. For each qualified DEmiRNA from independent comparisons, that was reported as binary dysregulation (i.e., upregulation or downregulation) in $\mathrm{AD}$ group compared with healthy control group. The effect sizes of binary data were calculated as $\log _{\mathrm{e}}$ odds ratios (logORs) based on the number of dysregulation events in both disease and control samples. The heterogeneity of each DEmiRNA was reported as tau square $\left(\tau^{2}\right)$ based on the restricted maximum-likelihood estimator and $I^{2}$ statistics. The outcomes were logORs with a 95\% confidence interval $(\mathrm{CI}), P$ values, $\mathrm{\tau}^{2}$, and $I^{2}$. For each DEmiRNA in the $i$ th study, the effect size $\left(\theta_{i}\right)$ based on the numbers of dysregulation events in both $\mathrm{AD}$ and control samples was calculated according to the formula, $\log \left(\frac{A_{i} * D_{i}}{B_{i} * C_{i}}\right)$, where $\mathrm{A}_{i}$ and $\mathrm{B}_{i}\left(\mathrm{C}_{i}\right.$ and $\left.\mathrm{D}_{i}\right)$ represent the number of upregulated and downregulated cases in the disease (control) group, respectively. Then the overall effect was computed according to formula, $\frac{\sum W_{i} \theta_{i}}{\sum W_{i}}$, where $\mathrm{W}_{i}$ is the weight and is equal to $1 /\left(\mathrm{v}_{i}+\mathrm{T}^{2}\right)$, where $\mathrm{v}_{i}$ is the sample variance. A larger sample size has more weight on the overall effect size. The $P$ values were adjusted by false discovery rate (FDR), and DEmiRNAs with FDRadjusted $P$ values less than 0.05 were regarded as statistically significant. Statistically significant DEmiRNAs with logORs above or below 0 were considered upregulated or downregulated, respectively, in $\mathrm{AD}$ compared with healthy controls.

Subgroup analysis was conducted based on the DEmiRNAs collected from the included differential miRNA expression studies. The DEmiRNAs were split into four different subgroups based on the blood sample sources, i.e., whole blood, plasma, serum, and peripheral blood mononuclear cell (PBMC).

\section{ABMDA identification of DEmiRNAs}

ABMDA ensemble learning was used to identify potential miRNA-disease associations. The original miRNAdisease associations with significant dysregulation in the "circulation_biomarker_diagnosis" category were extracted from HMDD, in which the associations are experimentally verified. DEmiRNAs identified in the metaanalysis with adjusted $P$ values less than 0.05 were input into the ABMDA ensemble learning method as positive miRNA-AD associations to further increase the number of positive miRNA-AD associations. In addition to initial miRNA-disease associations, ABMDA ensemble learning also requires disease-disease similarity and miRNA-miRNA functional similarity. The disease-disease similarity was determined using the DOSE [46] package for $\mathrm{R}$ software. The miRNA-miRNA functional similarity was retrieved from the database MISIM [47]. The ABMDA-identified DEmiRNAs were sorted by their prediction scores, and the DEmiRNAs with top prediction scores were collected until the first predicted DEmiRNA that was reported statistically insignificant in the meta-analysis. The names of predicted DEmiRNAs were standardized using the miRNAmeConverter package for $\mathrm{R}$ software with the miRBase database [17]. The ABMDA-identified DEmiRNAs with the top prediction scores, together with the meta-analysis DEmiRNAs were considered potential AD biomarkers.

\section{Biological significance of DEmiRNAs from meta-analysis and ABMDA by independent and synergic biological enrichment analysis}

Each miRNA regulates a large number of genes to exert a profound influence on genetic expression in specific cellular functions, and the primary function of each 
miRNA can be understood by identifying its target genes. The target genes of the DEmiRNAs from the meta-analysis and ABMDA identification were obtained using the multiMiR [48] package for $\mathrm{R}$ software based on the database miRTarBase [49]. The target genes of two DEmiRNA categories were collected separately to conduct two independent enrichment analyses and were also combined to conduct a synergic enrichment analysis. The biological enrichment analysis was conducted using the clusterProfiler [50] package for $\mathrm{R}$ software based on the Kyoto Encyclopedia of Genes and Genomes (KEGG) [51]. The pathways with FDR-adjusted $P$ values less than 0.05 were considered statistically significant.

\section{Collaborative biological function of DEmiRNAs from meta-analysis and ABMDA by network analysis}

The target gene semantic similarity measurements of the DEmiRNAs identified from the meta-analysis and ABMDA were computed using the DOSE package for $\mathrm{R}$. The target genes with semantic similarity over 0.95 were treated as common target genes in the two DEmiRNA categories. The DEmiRNAs and common target genes of the two DEmiRNA categories were used to construct a DEmiRNA-gene network based on the data from STRING (version 11) [52] to investigate the collaborative function of the DEmiRNAs identified from the meta-analysis and ABMDA. Only interactions with the highest confidence (0.9) were kept from the STRING.

\section{Risk of bias}

The risk of bias for each included study was evaluated according to the Minimum Information for Publication of Quantitative Real-time PCR Experiments (MIQE) [53]. This guideline is designed to assess the quality of qRT-PCR data and describes the minimum information required to ensure that experimental results can be comprehensively interpreted and independently verified. In the present study, the guideline was used to assess the quality of the expression profiling analysis of the included studies, including experimental design, sample annotation, experimental procedure, data processing pipeline, and result presentation. The evaluation was performed by two authors (SCY and XNL), independently. Disagreements between the authors were resolved by discussion with the other authors. Items with low risk were counted +1 , suggesting high reproducibility; items with unclear risk were counted 0 , suggesting ambiguous reproducibility; and items with high risk were counted -1 , suggesting low reproducibility.

\section{Results \\ Differential miRNA expression studies included in the analysis}

The selection process of the differential miRNA expression studies is shown in Fig. 2. A total of 7841 studies were initially identified from PubMed, ScienceDirect, and Web of Science. After a systematic search, 47 studies met the eligibility criteria and were included in the present study [23, 27-31, 39, 40, 54-92]. The characteristics of the included studies are shown in Table 1. The studies mainly focused on four blood elements: serum, plasma, whole blood, and peripheral blood mononuclear cells. Serum $(n=19)$ was the most extensively studied, followed by plasma $(n=16)$.

\section{Identification of DEmiRNAs from meta-analysis}

After DEmiRNA name standardization, there were 115 DEmiRNAs reported in 54 independent comparisons of 47 differential expression studies that compared AD blood samples with healthy blood samples. Eight-eight DEmiRNAs were found in one blood element, 25 DEmiRNAs were found in two blood elements, two DEmiRNAs were found in three blood elements, and no DEmiRNAs were found in all four blood elements. The most frequently reported DEmiRNAs in AD blood were miR-146a-5p and miR-26a-5p, which were dysregulated in five independent comparisons (Table 2). Of the 115 DEmiRNAs, 43 (37.4\%) were reported in at least two independent comparisons; dysregulation of 18 of them was reported consistently in the same direction, whereas dysregulation of 25 of them was reported in different directions. Based on the currently available data, none of the inconsistent DEmiRNA results were resolved in this meta-analysis. In the meta-analysis of 43 dysregulated miRNAs, 18 DEmiRNAs were found to be statistically significant (Table 3; Additional file 1); 7 and 11 DEmiRNAs were upregulated and downregulated, respectively. Among the 18 DEmiRNAs identified in this study, 6 of them (let-7d-5p, miR-107, miR-128-3p, miR-191-5p, miR-29c-3p, and miR-93-5p) were also found statistically significant in a previous meta-analysis [34]. The discrepancy could be due to that we only included the literature with qRT-PCR as validation results, and we treated comparisons independently even that those comparisons were from the same literature. Meanwhile, 13 DEmiRNAs of our meta-analytical results (let-7d-5p, miR-106b3p, miR-107, miR-126-5p, miR-148b-5p, miR-181c-3p, miR-191-5p, miR-200a-3p, miR-22-3p, miR-483-5p, miR-486-5p, miR-502-3p, and miR-93-5p), were reported contributing the AD diagnostic values in sensitivity and specificity in $\mathrm{Hu}$ et al. and Zhang et al. [35, 36]. The most significantly downregulated DEmiRNA was miR-107, which was identified in four independent comparisons. MiR-106b-39 was the most significantly 


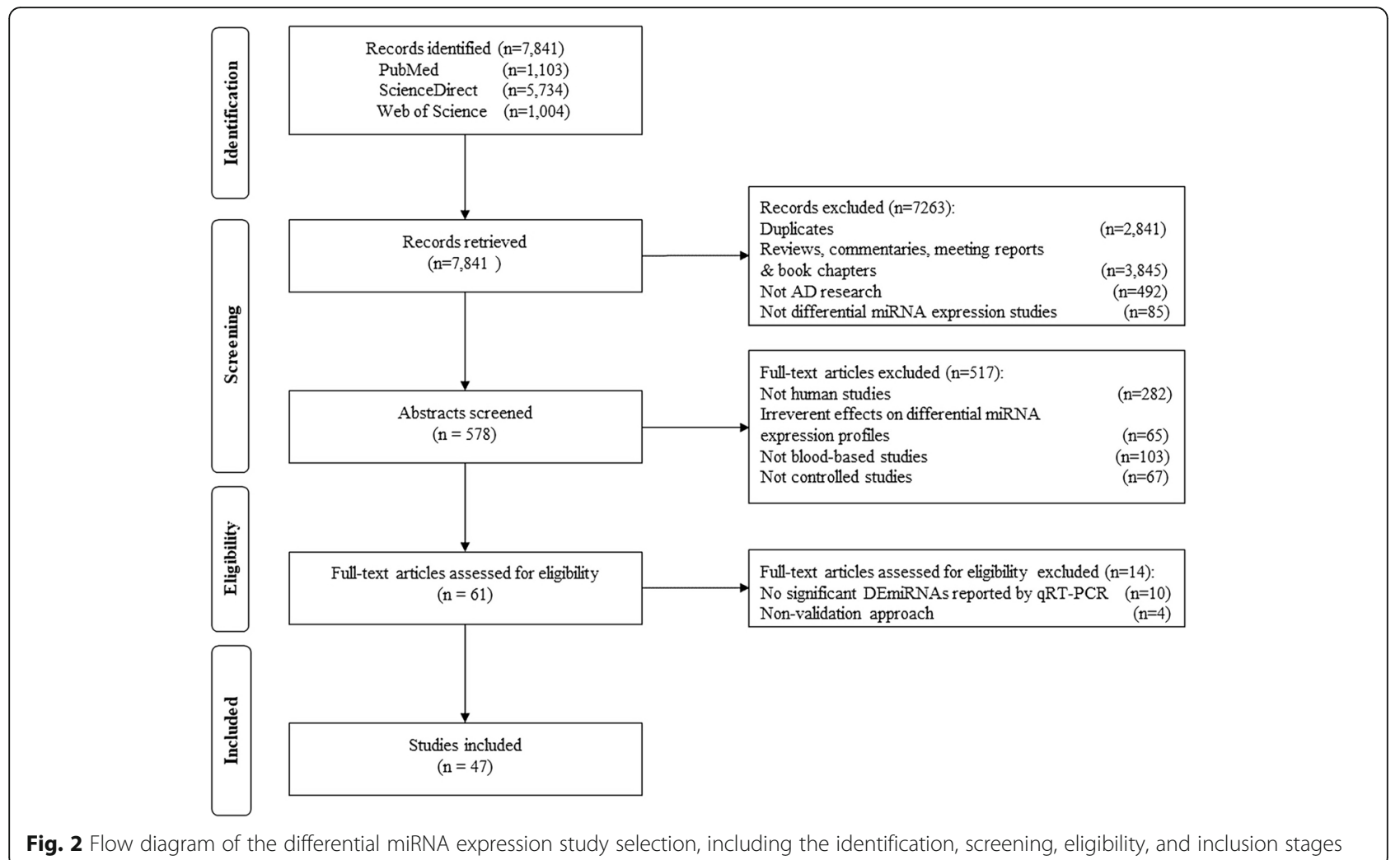

upregulated DEmiRNA among four independent comparisons. Downregulation of miR-107 has been reported to increase BACE1 expression [93] and influence cell cycle protein expression [94]. The dysregulation of miR106b-3p is negatively correlated with MMSE score [66] and modulates $A \beta$ metabolism [95]. Most DEmiRNAs identified in the meta-analysis were associated with mediating $A \beta$ generation, tau protein phosphorylation, and neuronal functions maintenance.

For subgroup analysis, among 54 comparisons, 7, 17, 23, and 7 comparisons investigated the DEmiRNAs in whole blood, plasma, serum, and $\mathrm{PBMC}$, respectively. The statistically significant DEmiRNAs from the subgroup meta-analysis are shown in Table 4. MiR-107 was consistently found in two subgroups, whole blood and plasma. Two miRNAs, miR-103a-3p in plasma and miR$181 c-5 p$ in serum, were found statistically significant in the subgroup analysis, but not in the meta-analysis. MiR-103a-3p is recently reported to be related to AD progression via regulating NPAS3 expression [96], while low level of miR-181c-5p in serum is suggested to be an indicator for cerebral vulnerability in $\mathrm{AD}$ [97].

\section{Identification of potential biomarkers by the ABMDA ensemble learning method}

A total of 1751 known miRNA-disease associations between 413 miRNAs and 227 diseases were obtained from the "circulation_biomarker_diagnosis" category in HMDD, including 17 known miRNA-AD associations. Identification of miRNA-disease associations by metaanalysis increased the number of positive miRNA-AD associations by 15 for the ABMDA identification after removing duplicates. The eleventh miRNA from the ABMDA results was determined to be statistically insignificant in the meta-analysis. Therefore, the first $10 \mathrm{miR}$ NAs with prediction scores ranging from 9.45 to 7.88 were collected (Table 5; Additional file 2). Most of these 10 miRNAs have been associated with multiple types of cancer as diagnostic or prognostic biomarkers [98-104]. Only one miRNA, miR-155, has been reported as a biomarker in AD for mediating neuroinflammation [105].

MiR-339-5p is upregulated to alleviate neuroinflammation by inhibiting HMGB1 [106]. HMGB1 encodes high mobility group box 1 to produce proinflammatory cytokines by binding to receptors for advanced glycation end products (RAGE) [107] and inhibiting IKK- $\beta$ and IKK- $\gamma$, which are key elements of NF- $\mathrm{kB}$ signaling [108]. $N F-\kappa B$ signaling can also be modulated by the identified DEmiRNAs through multiple mechanisms. Both miR203a-3p and let-7b-5p target IGF1R, which encodes insulin-like growth factor 1 receptor, to alleviate tumor necrosis factor (TNF)-induced activation of NF- $\mathrm{kB}$ [109, 110]. MiR-155 and miR-625-5p decrease the expression of SHIP and AKT2, which encode Src homology 2- 
Table 1 Characteristics of the included differential miRNA expression studies

\begin{tabular}{|c|c|c|c|c|c|c|c|}
\hline $\begin{array}{l}\text { PubMed } \\
\text { ID }\end{array}$ & Comparison ID & Blood elements & $\begin{array}{l}\text { Differential miRNA expression } \\
\text { profiling methods (screening } \\
\text { method/validation method) }\end{array}$ & $\begin{array}{l}\text { Number of } \\
\text { control case }\end{array}$ & $\begin{array}{l}\text { Number of } \\
A D \text { case }\end{array}$ & $\begin{array}{l}\text { Number of } \\
\text { upregulated } \\
\text { miRNA }\end{array}$ & $\begin{array}{l}\text { Number of } \\
\text { downregulated } \\
\text { miRNA }\end{array}$ \\
\hline 31857133 & 31857133_Ser & Serum & qRT-PCR & 93 & 108 & & 1 \\
\hline 31811079 & 31811079_Ser & Serum & qRT-PCR & 51 & 32 & 9 & 3 \\
\hline 31849573 & 31849573_Pla & Plasma & microRNA microarray/qRT-PCR & 31 & 16 & & 2 \\
\hline 31809862 & 31809862_WB & Whole blood & qRT-PCR & 214 & 145 & 1 & 1 \\
\hline 31766231 & 31766231_Pla & Plasma & microRNA microarray/qRT-PCR & 29 & 23 & 1 & \\
\hline 31691877 & 31691877_Ser & Serum & qRT-PCR & 30 & 30 & 4 & \\
\hline 31592314 & 31592314_Pla & Plasma & RNA deep-sequencing/qRT-PCR & 11 & 10 & & 6 \\
\hline 31572518 & 31572518_Ser & Serum & qRT-PCR & 98 & 105 & & 1 \\
\hline 31420923 & 31420923_Pla & Plasma & qRT-PCR & 120 & 120 & & 2 \\
\hline 31092279 & 31092279_Pla & Plasma & qRT-PCR & 14 & 56 & 3 & \\
\hline 30914454 & 30914454_Pla & Plasma & qRT-PCR & 385 & 385 & & 5 \\
\hline 30328325 & 30328325_Pla & Plasma & qRT-PCR & 20 & 20 & 1 & \\
\hline 29966198 & 29966198_Pla & Plasma & qRT-PCR & 10 & 10 & 2 & \\
\hline 29746584 & 29746584_Ser & Serum & qRT-PCR & 38 & 47 & 14 & 8 \\
\hline 29635818 & 29635818_Pla & Plasma & qRT-PCR & 20 & 20 & 1 & \\
\hline 29606187 & 29606187_Ser & Serum & qRT-PCR & 107 & 228 & 2 & 1 \\
\hline 29036829 & 29036829_WB & Whole blood & microRNA microarray/qRT-PCR & 17 & 21 & & 3 \\
\hline \multirow[t]{3}{*}{29036818} & $\begin{array}{l}\text { 29036818_Ser_ } \\
\text { Mild }\end{array}$ & Serum & RNA deep-sequencing/qRT-PCR & 86 & 31 & 1 & 5 \\
\hline & $\begin{array}{l}\text { 29036818_Ser_ } \\
\text { Mod }\end{array}$ & Serum & RNA deep-sequencing/qRT-PCR & 86 & 52 & 3 & 5 \\
\hline & $\begin{array}{l}\text { 29036818_Ser_ } \\
\text { Sev }\end{array}$ & Serum & RNA deep-sequencing/qRT-PCR & 86 & 38 & 4 & 5 \\
\hline 28934394 & 28934394_Ser & Serum & microRNA microarray/qRT-PCR & 18 & 11 & 1 & \\
\hline \multirow[t]{2}{*}{28849039} & $\begin{array}{l}\text { 28849039_Ser_ } \\
\text { Mil }\end{array}$ & Serum & microRNA microarray/qRT-PCR & 30 & 30 & & 1 \\
\hline & $\begin{array}{l}\text { 28849039_Ser_ } \\
\text { Mod }\end{array}$ & Serum & microRNA microarray/qRT-PCR & 30 & 30 & & 1 \\
\hline 28626163 & 28626163_Ser & Serum & RNA deep-sequencing/qRT-PCR & 40 & 45 & 5 & 4 \\
\hline \multirow[t]{2}{*}{28179587} & $\begin{array}{l}\text { 28179587_Pla_ } \\
\text { AD2 }\end{array}$ & Plasma & qRT-PCR & 9 & 13 & 4 & 3 \\
\hline & $\begin{array}{l}\text { 28179587_Pla_ } \\
\text { MCl-AD2 }\end{array}$ & Plasma & qRT-PCR & 9 & 8 & 4 & \\
\hline 28137310 & 28137310_Ser & Serum & RNA deep-sequencing/qRT-PCR & 22 & 36 & & 1 \\
\hline 27545218 & 27545218_Ser & Serum & qRT-PCR & 40 & 48 & 1 & \\
\hline 27501295 & 27501295_WB & Whole blood & qRT-PCR & 109 & 172 & & 4 \\
\hline 27446280 & 27446280_WB & Whole blood & qRT-PCR & 25 & 25 & & 1 \\
\hline 27277332 & 27277332_WB & Whole blood & qRT-PCR & 30 & 30 & 1 & \\
\hline 27239545 & 27239545_PBMC & $\begin{array}{l}\text { Peripheral blood } \\
\text { mononuclear cells }\end{array}$ & qRT-PCR & 36 & 36 & 3 & \\
\hline 27027823 & 27027823_Ser & Serum & qRT-PCR & 62 & 84 & 1 & 3 \\
\hline 26973465 & 26973465_Pla & Plasma & microRNA microarray/qRT-PCR & 40 & 40 & & 3 \\
\hline 26497032 & 26497032_PBMC & $\begin{array}{l}\text { Peripheral blood } \\
\text { mononuclear cells }\end{array}$ & microRNA microarray/qRT-PCR & 41 & 45 & 2 & 1 \\
\hline 26078483 & 26078483_Ser & Serum & RNA deep-sequencing/qRT-PCR & 75 & 79 & & 4 \\
\hline 25955795 & 25955795_WB & Whole blood & qRT-PCR & 30 & 30 & & 1 \\
\hline
\end{tabular}


Table 1 Characteristics of the included differential miRNA expression studies (Continued)

\begin{tabular}{|c|c|c|c|c|c|c|c|}
\hline $\begin{array}{l}\text { PubMed } \\
\text { ID }\end{array}$ & Comparison ID & Blood elements & $\begin{array}{l}\text { Differential miRNA expression } \\
\text { profiling methods (screening } \\
\text { method/validation method) }\end{array}$ & $\begin{array}{l}\text { Number of } \\
\text { control case }\end{array}$ & $\begin{array}{l}\text { Number of } \\
\text { AD case }\end{array}$ & $\begin{array}{l}\text { Number of } \\
\text { upregulated } \\
\text { miRNA }\end{array}$ & $\begin{array}{l}\text { Number of } \\
\text { downregulated } \\
\text { miRNA }\end{array}$ \\
\hline 25742200 & 25742200_Pla & Plasma & qRT-PCR & 81 & 97 & & 1 \\
\hline 25667669 & 25667669_Ser & Serum & qRT-PCR & 42 & 26 & & 1 \\
\hline 25152461 & 25152461_Ser & Serum & microRNA microarray/qRT-PCR & 30 & 38 & & 2 \\
\hline \multirow[t]{2}{*}{24827165} & 24827165_Pla & Plasma & qRT-PCR & 7 & 7 & & 1 \\
\hline & 24827165_Ser & Serum & qRT-PCR & 7 & 7 & & 1 \\
\hline 24577456 & 24577456_Ser & Serum & RNA deep-sequencing/qRT-PCR & 155 & 158 & & 6 \\
\hline \multirow[t]{2}{*}{24550773} & 24550773_Pla & Plasma & qRT-PCR & 27 & 25 & 1 & \\
\hline & 24550773_PBMC & $\begin{array}{l}\text { Peripheral blood } \\
\text { mononuclear cells }\end{array}$ & qRT-PCR & 27 & 25 & 1 & \\
\hline 24157723 & 24157723_Pla & Plasma & qRT-PCR & 10 & 10 & & 2 \\
\hline 24139697 & 24139697_Ser & Serum & qRT-PCR & 155 & 105 & 1 & 2 \\
\hline \multirow[t]{2}{*}{24064186} & $\begin{array}{l}\text { 24064186_ } \\
\text { PBMC_Mon }\end{array}$ & Monocytes & qRT-PCR & 37 & 34 & 1 & \\
\hline & $\begin{array}{l}\text { 24064186_ } \\
\text { PBMC_Lym }\end{array}$ & Lymphocytes & qRT-PCR & 37 & 34 & 1 & \\
\hline 23922807 & $\begin{array}{l}\text { 23922807_Pla } \\
\text { C2 }\end{array}$ & Plasma & microRNA microarray/qRT-PCR & 17 & 20 & & 6 \\
\hline 23895045 & 23895045_WB & Whole blood & RNA deep-sequencing/qRT-PCR & 21 & 94 & 5 & 7 \\
\hline 23435408 & 23435408_PBMC & $\begin{array}{l}\text { Peripheral blood } \\
\text { mononuclear cells }\end{array}$ & qRT-PCR & 25 & 28 & & 1 \\
\hline 22155483 & 22155483_Ser & Serum & qRT-PCR & 7 & 7 & & 5 \\
\hline 19936094 & 19936094_PBMC & $\begin{array}{l}\text { Peripheral blood } \\
\text { mononuclear cells }\end{array}$ & microRNA microarray/qRT-PCR & 5 & 5 & 2 & \\
\hline
\end{tabular}

containing inositol phosphatase and RAC-beta serine/ threonine-protein kinase, respectively, to attenuate NFкB-dependent inflammation [111, 112]. Additionally, miR-495 targets NOD1, which encodes nucleotidebinding oligomerization domain-containing protein 1 , to reduce high glucose-induced inflammation in diabetic complications [113], whereas its mature form, miR-495$3 \mathrm{p}$, has recently been reported to regulate inflammatory molecules by targeting IL5RA [114]. The mature forms of miR-128-2, let-7a-2, miR-103a-2, and miR-16-2 have not been reported in the miRBase database at the time of this study; however, their family members exhibit inflammatory properties. MiR-128 and let-7a regulate gene expression in response to oxidative stress [115, 116]. MiR-16 and miR-103-3p target ADORA2A and SNRK, which encode the adenosine A2a receptor and sucrose non-fermentable-related serine/threonine-protein kinase, respectively, to attenuate $\mathrm{NF}-\mathrm{kB}$-dependent inflammation $[117,118]$. Current literature suggests that NF-kB might be the main downstream effector for the DEmiRNAs identified by ABMDA in the present study.

\section{Enrichment analysis}

There were 3496 and 2938 target genes in total for 18 and 10 DEmiRNAs from the meta-analysis and ABMDA results, respectively (Additional file 3). These target genes were subjected to two independent enrichment analyses based on KEGG to obtain the functional annotations of the DEmiRNAs (Fig. 3A, B). Most pathways targeted by the meta-analysis and ABMDA DEmiRNAs were commonly found in the two independent enrichment analyses, even though the DEmiRNAs in the two categories were not identical. This indicated that multiple DEmiRNAs target either common or different mRNA transcripts that functionally converge on the same pathways. Most dysregulated pathways in the enrichment analysis were involved in AD development via modulation of neuroinflammation, including the AGERAGE signaling pathway in diabetic complications, cell cycle, cellular senescence, Hippo signaling pathway, and FoxO signaling pathways. In addition, multiple cancerous pathways were also implicated. Compared with the two independent enrichment analyses, the synergic enrichment analysis unifying the two categories of DEmiRNAs might provide better insight for AD development. In the synergic enrichment analysis, the dysregulated pathways were more statistically significant (Fig. 3C). Also, the pathways that were found in only one independent enrichment analysis (such as the Hippo signaling pathway from the meta-analysis and cell cycle from 
Table 2 DEmiRNAs reported by the included differential miRNA expression studies. The bold DEmiRNAs were reported by at least two independent comparisons and were qualified for the subsequent meta-analysis

\begin{tabular}{|c|c|c|c|}
\hline Comparison ID & MiRNAs & Dysregulated direction & $P$ value \\
\hline 31857133_Ser & miR-193a-3p & Down & $<0.001$ \\
\hline \multirow[t]{11}{*}{ 31811079_Ser } & $\operatorname{miR}-346$ & Up & 0.0013 \\
\hline & miR-345-5p & Up & 0.0239 \\
\hline & miR-122-3p & Up & 0.0001 \\
\hline & miR-1291 & Up & 0.0052 \\
\hline & miR-640 & Up & 0.0004 \\
\hline & miR-650 & Up & 0.0035 \\
\hline & miR-1285-3p & Up & 0.0032 \\
\hline & miR-1299 & Up & 0.0003 \\
\hline & miR-1267 & Up & 0.0055 \\
\hline & miR-208b-3p & Down & 0.0006 \\
\hline & miR-206 & Down & 0.0004 \\
\hline \multirow[t]{2}{*}{ 31849573_Pla } & miR-132-3p & Down & 0.0333 \\
\hline & miR-212-3p & Down & 0.001 \\
\hline \multirow[t]{2}{*}{ 31809862_WB } & miR-532-5p & Up & $4.8 \times 10 E-30$ \\
\hline & miR-1468-5p & Down & $6.2 \times 10 E-12$ \\
\hline 31766231_Pla & miR-206 & Up & $<0.025$ \\
\hline \multirow[t]{4}{*}{ 31691877_Ser } & $\operatorname{miR}-22-5 p$ & Up & $\leq 0.005$ \\
\hline & miR-23a-3p & Up & $\leq 0.05$ \\
\hline & miR-29a-3p & Up & $\leq 0.05$ \\
\hline & miR-125b-5p & Up & $\leq 0.005$ \\
\hline \multirow[t]{6}{*}{ 31592314_Pla } & miR-451a & Down & $<0.0005$ \\
\hline & $\operatorname{miR}-21-5 p$ & Down & $<0.005$ \\
\hline & miR-23a-3p & Down & $<0.005$ \\
\hline & let-7i-5p & Down & $<0.05$ \\
\hline & miR-126-3p & Down & $<0.005$ \\
\hline & miR-151a-3p & Down & $<0.05$ \\
\hline 31572518_Ser & miR-133b & Down & $<0.001$ \\
\hline \multirow[t]{2}{*}{ 31420923_Pla } & miR-103a-3p & Down & $<0.001$ \\
\hline & miR-107 & Down & $<0.001$ \\
\hline \multirow[t]{3}{*}{ 31092279_Pla } & miR-92a-3p & Up & 0.0442 \\
\hline & miR-181c-5p & Up & 0.0024 \\
\hline & miR-210-3p & Up & 0.0006 \\
\hline \multirow[t]{5}{*}{ 30914454_Pla } & miR-101-3p & Down & $<0.001$ \\
\hline & miR-153-3p & Down & $<0.001$ \\
\hline & miR-144-3p & Down & $<0.001$ \\
\hline & miR-381-3p & Down & $<0.001$ \\
\hline & miR-383-5p & Down & $<0.001$ \\
\hline 30328325_Pla & miR-128-3p & Up & $<0.05$ \\
\hline \multirow[t]{2}{*}{ 29966198_Pla } & miR-146a-5p & Up & $<0.05$ \\
\hline & miR-933 & Up & $<0.05$ \\
\hline \multirow[t]{2}{*}{ 29746584_Ser } & miR-103a-3p & Up & $<0.05$ \\
\hline & miR-142-3p & Up & $<0.05$ \\
\hline
\end{tabular}


Table 2 DEmiRNAs reported by the included differential miRNA expression studies. The bold DEmiRNAs were reported by at least two independent comparisons and were qualified for the subsequent meta-analysis (Continued)

\begin{tabular}{|c|c|c|c|}
\hline Comparison ID & MiRNAs & Dysregulated direction & $P$ value \\
\hline & miR-20a-5p & Up & $<0.05$ \\
\hline & miR-29b-3p & Up & $<0.05$ \\
\hline & let-7b-5p & Up & $<0.05$ \\
\hline & let-7 g-5p & Up & $<0.05$ \\
\hline & miR-106a-5p & Up & $<0.05$ \\
\hline & miR-106b-5p & Up & $<0.05$ \\
\hline & miR-18b-5p & Up & $<0.05$ \\
\hline & miR-223-3p & Up & $<0.05$ \\
\hline & miR-26a-5p & Up & $<0.05$ \\
\hline & miR-26b-5p & Up & $<0.05$ \\
\hline & miR-301a-3p & Up & $<0.05$ \\
\hline & miR-30b-5p & Up & $<0.05$ \\
\hline & miR-132-3p & Down & $<0.05$ \\
\hline & miR-146a-5p & Down & $<0.05$ \\
\hline & miR-15a-5p & Down & $<0.05$ \\
\hline & miR-22-3p & Down & $<0.05$ \\
\hline & miR-320a-3p & Down & $<0.05$ \\
\hline & miR-320b & Down & $<0.05$ \\
\hline & miR-92a-3p & Down & $<0.05$ \\
\hline & miR-1246 & Down & $<0.05$ \\
\hline 29635818_Pla & miR-1908-5p & Up & $<0.05$ \\
\hline \multirow[t]{3}{*}{ 29606187_Ser } & miR-135a-5p & Up & $<0.05$ \\
\hline & miR-193b-3p & Down & $<0.01$ \\
\hline & miR-384 & Up & $<0.05$ \\
\hline \multirow[t]{3}{*}{ 29036829_WB } & miR-144-5p & Down & 0.03 \\
\hline & miR-374a-5p & Down & 0.034 \\
\hline & miR-221-3p & Down & 0.042 \\
\hline \multirow[t]{6}{*}{ 29036818_Ser_Mild } & miR-106b-3p & Up & $<0.001$ \\
\hline & miR-26a-5p & Down & $<0.001$ \\
\hline & miR-181c-3p & Down & $<0.001$ \\
\hline & miR-126-5p & Down & $<0.001$ \\
\hline & miR-22-3p & Down & $<0.001$ \\
\hline & miR-148b-5p & Down & $<0.001$ \\
\hline \multirow[t]{7}{*}{ 29036818_Ser_Mod } & miR-106b-3p & Up & $<0.001$ \\
\hline & miR-1246 & Up & $<0.001$ \\
\hline & miR-26a-5p & Down & $<0.001$ \\
\hline & miR-181c-3p & Down & $<0.001$ \\
\hline & miR-126-5p & Down & $<0.001$ \\
\hline & miR-22-3p & Down & $<0.001$ \\
\hline & miR-148b-5p & Down & $<0.001$ \\
\hline \multirow[t]{3}{*}{ 29036818_Ser_Sev } & miR-106b-3p & Up & $<0.001$ \\
\hline & miR-1246 & Up & $<0.001$ \\
\hline & miR-660-5p & Up & $<0.001$ \\
\hline
\end{tabular}


Table 2 DEmiRNAs reported by the included differential miRNA expression studies. The bold DEmiRNAs were reported by at least two independent comparisons and were qualified for the subsequent meta-analysis (Continued)

\begin{tabular}{|c|c|c|c|}
\hline Comparison ID & MiRNAs & Dysregulated direction & $P$ value \\
\hline & miR-26a-5p & Down & 0.007 \\
\hline & miR-181c-3p & Down & $<0.001$ \\
\hline & miR-126-5p & Down & $<0.001$ \\
\hline & miR-22-3p & Down & $<0.001$ \\
\hline & $\operatorname{miR}-148 b-5 p$ & Down & $<0.001$ \\
\hline 28934394_Ser & miR-455-3p & Up & 0.007 \\
\hline 28849039_Ser_Mil & miR-222-3p & Down & $<0.05$ \\
\hline 28849039_Ser_Mod & miR-222-3p & Down & $<0.05$ \\
\hline \multirow[t]{9}{*}{ 28626163_Ser } & miR-146a-5p & Up & $<0.05$ \\
\hline & miR-106b-3p & Up & $<0.05$ \\
\hline & miR-195-5p & Up & $<0.05$ \\
\hline & miR-20b-5p & Up & $<0.05$ \\
\hline & miR-497-5p & Up & $<0.05$ \\
\hline & miR-29c-3p & Down & $<0.05$ \\
\hline & miR-93-5p & Down & $<0.05$ \\
\hline & miR-19b-3p & Down & $<0.05$ \\
\hline & miR-125b-3p & Down & $<0.05$ \\
\hline \multirow[t]{7}{*}{ 28179587_Pla_AD2 } & miR-486-5p & Up & $<0.001$ \\
\hline & miR-483-5p & Up & $<0.0001$ \\
\hline & miR-502-3p & Up & $<0.0001$ \\
\hline & miR-200a-3p & Up & $<0.01$ \\
\hline & miR-151a-5p & Down & $<0.001$ \\
\hline & miR-30b-5p & Down & $<0.01$ \\
\hline & miR-103a-3p & Down & $<0.01$ \\
\hline \multirow[t]{4}{*}{ 28179587_Pla_MCl-AD2 } & miR-486-5p & Up & $<0.001$ \\
\hline & miR-483-5p & Up & $<0.001$ \\
\hline & miR-502-3p & Up & $<0.01$ \\
\hline & miR-200a-3p & Up & $<0.05$ \\
\hline 28137310_Ser & miR-501-3p & Down & 0.002 \\
\hline 27545218_Ser & miR-613 & Up & $<0.01$ \\
\hline \multirow[t]{4}{*}{ 27501295_WB } & miR-9-5p & Down & 0.001 \\
\hline & miR-106a-5p & Down & 0.001 \\
\hline & miR-106b-5p & Down & 0.008 \\
\hline & miR-107 & Down & 0.001 \\
\hline 27446280_WB & $\operatorname{miR}-135 b-5 p$ & Down & $<0.01$ \\
\hline 27277332_WB & miR-206 & Up & $<0.001$ \\
\hline \multirow[t]{3}{*}{ 27239545_PBMC } & miR-27b-3p & Up & $<0.05$ \\
\hline & miR-128-3p & Up & $<0.05$ \\
\hline & miR-155-5p & Up & $<0.05$ \\
\hline \multirow[t]{4}{*}{ 27027823_Ser } & miR-125b-5p & Down & $<0.001$ \\
\hline & miR-223-3p & Down & $<0.001$ \\
\hline & miR-29 & Down & $<0.01$ \\
\hline & miR-519 & Up & $<0.001$ \\
\hline
\end{tabular}


Table 2 DEmiRNAs reported by the included differential miRNA expression studies. The bold DEmiRNAs were reported by at least two independent comparisons and were qualified for the subsequent meta-analysis (Continued)

\begin{tabular}{|c|c|c|c|}
\hline Comparison ID & MiRNAs & Dysregulated direction & $P$ value \\
\hline \multirow[t]{3}{*}{ 26973465_Pla } & miR-10b-5p & Down & 0.022 \\
\hline & miR-29a-3p & Down & 0.041 \\
\hline & miR-130b-3p & Down & 0.002 \\
\hline \multirow[t]{3}{*}{ 26497032_PBMC } & miR-425-5p & Up & $<0.001$ \\
\hline & miR-339-5p & Up & 0.003 \\
\hline & miR-639 & Down & 0.04 \\
\hline \multirow[t]{4}{*}{ 26078483_Ser } & miR-31-5p & Down & $<0.0001$ \\
\hline & miR-93-5p & Down & $<0.0001$ \\
\hline & miR-143-3p & Down & $<0.0001$ \\
\hline & miR-146a-5p & Down & $<0.0001$ \\
\hline 25955795_WB & miR-29c-3p & Down & 0.0001 \\
\hline 25742200_Pla & miR-107 & Down & $<0.001$ \\
\hline 25667669_Ser & miR-210-3p & Down & $<0.01$ \\
\hline \multirow[t]{2}{*}{ 25152461_Ser } & miR-135a-5p & Down & $<0.05$ \\
\hline & miR-200b-3p & Down & $<0.05$ \\
\hline 24827165_Pla & miR-384 & Down & $<0.05$ \\
\hline 24827165_Ser & miR-384 & Down & $<0.05$ \\
\hline \multirow[t]{6}{*}{ 24577456_Ser } & miR-98-5p & Down & $2.67 \times 10 E-4$ \\
\hline & miR-885-5p & Down & $2.8 \times 10 \mathrm{E}-4$ \\
\hline & miR-483-3p & Down & $1.0 \times 10 E-4$ \\
\hline & miR-342-3p & Down & $9.19 \times 10 E-16$ \\
\hline & miR-191-5p & Down & $1.54 \times 10 E-9$ \\
\hline & let-7d-5p & Down & $1.2 \times 10 \mathrm{E}-6$ \\
\hline 24550773_Pla & miR-34c-5p & Up & $<0.01$ \\
\hline 24550773_PBMC & miR-34c-5p & Up & $<0.01$ \\
\hline \multirow[t]{2}{*}{ 24157723_Pla } & miR-34a-5p & Down & $<0.05$ \\
\hline & miR-146a-5p & Down & $<0.05$ \\
\hline \multirow[t]{3}{*}{ 24139697_Ser } & miR-125b-5p & Down & $<0.0001$ \\
\hline & miR-181c-5p & Down & $<0.0001$ \\
\hline & miR-9-5p & Up & 0.0045 \\
\hline 24064186_PBMC_Mon & miR-128-3p & Up & $<0.05$ \\
\hline 24064186_PBMC_Lym & miR-128-3p & Up & $<0.05$ \\
\hline \multirow[t]{6}{*}{ 23922807_Pla_C2 } & let-7d-5p & Down & 0.0001 \\
\hline & let-7 g-5p & Down & 0.001 \\
\hline & miR-15b-5p & Down & 0.001 \\
\hline & miR-142-3p & Down & 0.0001 \\
\hline & miR-191-5p & Down & 0.002 \\
\hline & miR-545-3p & Down & 0.03 \\
\hline \multirow[t]{5}{*}{ 23895045_WB } & miR-151a-3p & Up & $<0.05$ \\
\hline & let-7d-3p & Up & $<0.05$ \\
\hline & miR-5010-3p & Up & $<0.05$ \\
\hline & let-7f-5p & Down & $<0.05$ \\
\hline & miR-1285-5p & Down & $<0.05$ \\
\hline
\end{tabular}


Table 2 DEmiRNAs reported by the included differential miRNA expression studies. The bold DEmiRNAs were reported by at least two independent comparisons and were qualified for the subsequent meta-analysis (Continued)

\begin{tabular}{|c|c|c|c|}
\hline Comparison ID & MiRNAs & Dysregulated direction & $P$ value \\
\hline & miR-107 & Down & $<0.05$ \\
\hline & miR-103a-3p & Down & $<0.05$ \\
\hline & miR-26b-5p & Down & $<0.05$ \\
\hline & miR-26a-5p & Down & $<0.05$ \\
\hline & miR-532-5p & Down & $<0.05$ \\
\hline 23435408_PBMC & miR-29b-3p & Down & 0.002 \\
\hline \multirow[t]{5}{*}{ 22155483_Ser } & miR-137-3p & Down & $<0.05$ \\
\hline & miR-181c-5p & Down & $<0.05$ \\
\hline & miR-9-5p & Down & $<0.05$ \\
\hline & miR-29a-3p & Down & $<0.05$ \\
\hline & miR-29b-3p & Down & $<0.05$ \\
\hline \multirow[t]{2}{*}{ 19936094_PBMC } & $\operatorname{miR}-34 a-5 p$ & Up & $<0.05$ \\
\hline & miR-181b-5p & Up & $<0.05$ \\
\hline
\end{tabular}

ABMDA) were both statistically significant in the synergic enrichment analysis. The synergic enrichment analysis suggested that the two DEmiRNA categories interact functionally and complement each other.

In the synergic enrichment analysis, the AGE-RAGE signaling pathway in diabetic complications was identified as the second most statistically significant. The AGE-RAGE pathway is involved in diabetic microvascular complications. Elevated levels of AGE and RAGE have also been reported in AD patients, and increased RAGE activity has been detected in patients with early $\mathrm{AD}$ symptoms $[119,120]$. RAGE also interacts with $\mathrm{A} \beta$ oligomers to induce BBB leakage [121] and upregulates NF- $\mathrm{kB}$, which induces neuroinflammation [122]. Elevated neuroinflammation increases the expression of secretases for $A \beta$ production [123], reduces $A \beta$ degradation in microglia [124], and induces the aberrant hyperphosphorylation of tau proteins [125]. Elevated levels of proinflammatory cytokines and $\mathrm{A} \beta$ increase cell cyclerelated kinases, such as PKA, CAMKII, Fyn, and mTORC1, inducing neuronal cell cycle re-entry (CCR) $[126,127]$. Aberrant CCR results in neuronal hyperploidy, which alters neuronal circuit function and reduces synaptic activity [128, 129], ultimately inducing neuronal death [130]. Aberrant CCR is also induced by malfunction of $\mathrm{PI} 3 \mathrm{~K} / \mathrm{AKT} / \mathrm{mTOR}$, a cell survival pathway disrupted in both $\mathrm{AD}$ and cancer, though in opposite directions [131]. Further, aberrant CCR is a causative factor for the majority of neuronal death in early AD development and might be a potential biological mechanism to link $\mathrm{AD}$ and multiple cancerous diseases. Aberrant $A \beta$ accumulation and proinflammatory cytokines from dying neurons further enhance neuroinflammation and oxidative stress, inducing cellular senescence and dysregulating the Hippo and FoxO signaling pathways. Cellular senescence is a permanent state of cellular rest that is involved in the onset of $\mathrm{AD}$ [132], and neuroinflammation and $A \beta$-mediated toxicity have been reported to upregulate senescence-regulated genes [133135]. The Hippo signaling pathway is a kinase cascade relevant for cellular homeostasis, and is upregulated by $\mathrm{A} \beta$-mediated neurotoxicity to enhance neurodegeneration with JNK in AD [136, 137]. The FoxO signaling pathway is involved in the relationship between ROS, insulin resistance, and AD pathology [138, 139]. Under persistent oxidative stress, the FoxO signaling pathway increases the transcription of apoptotic proteins [140].

\section{Network}

In total, 5222 target genes were identified for the DEmiRNAs from the meta-analysis and ABMDA, and 1865 target genes with semantic similarity over 0.95 were identified as common target genes in the two DEmiRNA categories, suggesting an overlap of the two categories in biological functions. The common target genes were used to retrieve the corresponding proteinprotein interactions according to STRING and construct a network. The network comprised 1865 common target genes as nodes, and 18750 edges among the common target genes. The DEmiRNAs let-7b-5p and miR-155 identified by ABMDA shared the most common target genes with the DEmiRNAs miR-93-5p and miR-128-3p identified by the meta-analysis. The common target genes $U B C, U B B$, and $R P S 27 A$, which are core members in the ubiquitin-proteasome system (UPS), exhibited the highest connection degree in the network.

The UPS is imperative not just in A $\beta$ clearance [141], but also in neuroinflammation and neuronal CCR. The 
Table 3 Statistically significant DEmiRNAs identified by the meta-analysis

\begin{tabular}{|c|c|c|c|c|c|c|c|c|c|c|c|}
\hline MiRNAs & Comparison ID & $\begin{array}{l}\text { Number of } \\
\text { upregulated } \\
\text { case in } A D\end{array}$ & $\begin{array}{l}\text { Number of } \\
\text { downregulated } \\
\text { case in AD }\end{array}$ & $\begin{array}{l}\text { Number of } \\
\text { control case }\end{array}$ & Weight & $P$ value & FDR & LogOR & $95 \% \mathrm{Cl}$ & $\tau^{\wedge} 2$ & $1 \wedge 2$ \\
\hline \multirow[t]{4}{*}{ miR-107 } & 27501295_WB & & 172 & 109 & $25.08 \%$ & $3.74 \mathrm{E}-25$ & $1.61 E-23$ & -10.40 & {$[-12.36,-8.43]$} & 0.00 & $0.00 \%$ \\
\hline & 25742200_Pla & & 97 & 81 & $25.03 \%$ & & & & & & \\
\hline & 23895045_WB & & 94 & 21 & $24.82 \%$ & & & & & & \\
\hline & 31420923_Pla & & 120 & 120 & $25.07 \%$ & & & & & & \\
\hline \multirow[t]{4}{*}{ miR-106b-3p } & 29036818_Ser_Mild & 31 & & 86 & $24.98 \%$ & $1.05 E-20$ & $1.53 \mathrm{E}-19$ & 9.38 & {$[7.41,11.35]$} & 0.00 & $0.00 \%$ \\
\hline & 29036818_Ser_Mod & 52 & & 86 & $25.06 \%$ & & & & & & \\
\hline & 29036818_Ser_Sev & 38 & & 86 & $25.01 \%$ & & & & & & \\
\hline & 28626163_Ser & 45 & & 40 & $24.96 \%$ & & & & & & \\
\hline \multirow[t]{4}{*}{ miR-22-3p } & 29036818_Ser_Mild & & 31 & 86 & $24.98 \%$ & $1.07 \mathrm{E}-20$ & $1.53 \mathrm{E}-19$ & -9.38 & {$[-11.34,-7.41]$} & 0.00 & $0.00 \%$ \\
\hline & 29036818_Ser_Mod & & 52 & 86 & $25.06 \%$ & & & & & & \\
\hline & 29036818_Ser_Sev & & 38 & 86 & $25.01 \%$ & & & & & & \\
\hline & 29746584_Ser & & 47 & 38 & $24.95 \%$ & & & & & & \\
\hline \multirow[t]{3}{*}{ miR-126-5p } & 29036818_Ser_Mild & & 31 & 86 & $33.28 \%$ & $2.06 \mathrm{E}-16$ & $1.48 \mathrm{E}-15$ & -9.53 & {$[-11.81,-7.26]$} & 0.00 & $0.00 \%$ \\
\hline & 29036818_Ser_Mod & & 52 & 86 & $33.39 \%$ & & & & & & \\
\hline & 29036818_Ser_Sev & & 38 & 86 & $33.33 \%$ & & & & & & \\
\hline \multirow[t]{3}{*}{ miR-148b-5p } & 29036818_Ser_Mild & & 31 & 86 & $33.28 \%$ & $2.06 \mathrm{E}-16$ & $1.48 \mathrm{E}-15$ & -9.53 & {$[-11.81,-7.26]$} & 0.00 & $0.00 \%$ \\
\hline & 29036818_Ser_Mod & & 52 & 86 & $33.39 \%$ & & & & & & \\
\hline & 29036818_Ser_Sev & & 38 & 86 & $33.33 \%$ & & & & & & \\
\hline \multirow[t]{3}{*}{ miR-181c-3p } & 29036818_Ser_Mild & & 31 & 86 & $33.28 \%$ & $2.06 \mathrm{E}-16$ & $1.48 E-15$ & -9.53 & {$[-11.81,-7.26]$} & 0.00 & $0.00 \%$ \\
\hline & 29036818_Ser_Mod & & 52 & 86 & $33.39 \%$ & & & & & & \\
\hline & 29036818_Ser_Sev & & 38 & 86 & $33.33 \%$ & & & & & & \\
\hline \multirow[t]{4}{*}{ miR-128-3p } & 27239545_PBMC & 30 & & 30 & $25.02 \%$ & $4.64 E-16$ & $2.85 E-15$ & 8.19 & {$[6.21,10.17]$} & 0.00 & $0.00 \%$ \\
\hline & 24064186_PBMC_Mon & 34 & & 37 & $25.08 \%$ & & & & & & \\
\hline & 24064186_PBMC_Lym & 34 & & 37 & $25.08 \%$ & & & & & & \\
\hline & 30328325_Pla & 20 & & 20 & $24.82 \%$ & & & & & & \\
\hline \multirow[t]{2}{*}{ miR-93-5p } & 26078483_Ser & & 79 & 75 & $50.13 \%$ & $2.3 \mathrm{E}-11$ & $1.24 E-10$ & -9.50 & {$[-12.28,-6.71]$} & 0.00 & $0.00 \%$ \\
\hline & 28626163_Ser & & 45 & 40 & $49.87 \%$ & & & & & & \\
\hline \multirow[t]{2}{*}{$\operatorname{miR}-29 c-3 p$} & 25955795_WB & & 30 & 30 & $49.88 \%$ & $1.81 \mathrm{E}-09$ & 8.65E-09 & -8.56 & {$[-11.36,-5.77]$} & 0.00 & $0.00 \%$ \\
\hline & 28626163_Ser & & 45 & 40 & $50.12 \%$ & & & & & & \\
\hline \multirow[t]{2}{*}{ miR-132-3p } & 29746584_Ser & & 47 & 38 & $50.28 \%$ & $6.67 \mathrm{E}-09$ & $2.87 E-08$ & -8.27 & {$[-11.07,-5.48]$} & 0.00 & $0.00 \%$ \\
\hline & 31849573_Pla & & 16 & 31 & $49.72 \%$ & & & & & & \\
\hline \multirow[t]{2}{*}{ miR-222-3p } & 28849039_Ser_Mil & & 30 & 30 & $50.00 \%$ & $8.09 \mathrm{E}-09$ & $3.16 \mathrm{E}-08$ & -8.22 & {$[-11.02,-5.43]$} & 0.00 & $0.00 \%$ \\
\hline & 28849039_Ser_Mod & & 30 & 30 & $50.00 \%$ & & & & & & \\
\hline \multirow[t]{2}{*}{$\operatorname{miR}-34 c-5 p$} & 24550773_Pla & 25 & & 27 & $50.00 \%$ & $2.67 \mathrm{E}-08$ & $9.58 \mathrm{E}-08$ & 7.94 & {$[5.14,10.74]$} & 0.00 & $0.00 \%$ \\
\hline & 24550773_PBMC & 25 & & 27 & $50.00 \%$ & & & & & & \\
\hline \multirow[t]{2}{*}{ let-7d-5p } & 23922807_Pla_C2 & & 20 & 17 & $49.74 \%$ & $8.9 \mathrm{E}-06$ & 2.73E-05 & -9.39 & {$[-13.54,-5.25]$} & 4.89 & $54.62 \%$ \\
\hline & 24577456_Ser & & 158 & 155 & $50.26 \%$ & & & & & & \\
\hline \multirow[t]{2}{*}{ miR-191-5p } & 23922807_Pla_C2 & & 20 & 17 & $49.74 \%$ & $8.9 \mathrm{E}-06$ & 2.73E-05 & -9.39 & {$[-13.54,-5.25]$} & 4.89 & $54.62 \%$ \\
\hline & 24577456_Ser & & 158 & 155 & $50.26 \%$ & & & & & & \\
\hline \multirow[t]{2}{*}{ miR-200a-3p } & 28179587_Pla_AD2 & 13 & & 9 & $50.26 \%$ & 3.37E-05 & 8.05E-05 & 6.01 & {$[3.17,8.85]$} & 0.00 & $0.00 \%$ \\
\hline & 28179587_Pla_MCI-AD2 & 8 & & 9 & $49.74 \%$ & & & & & & \\
\hline \multirow[t]{2}{*}{ miR-483-5p } & 28179587_Pla_AD2 & 13 & & 9 & $50.26 \%$ & 3.37E-05 & $8.05 E-05$ & 6.01 & {$[3.17,8.85]$} & 0.00 & $0.00 \%$ \\
\hline & 28179587_Pla_MCI-AD2 & 8 & & 9 & $49.74 \%$ & & & & & & \\
\hline \multirow[t]{2}{*}{ miR-486-5p } & 28179587_Pla_AD2 & 13 & & 9 & $50.26 \%$ & 3.37E-05 & 8.05E-05 & 6.01 & {$[3.17,8.85]$} & 0.00 & $0.00 \%$ \\
\hline & 28179587_Pla_MCI-AD2 & 8 & & 9 & $49.74 \%$ & & & & & & \\
\hline \multirow[t]{2}{*}{ miR-502-3p } & 28179587_Pla_AD2 & 13 & & 9 & $50.26 \%$ & 3.37E-05 & 8.05E-05 & 6.01 & {$[3.17,8.85]$} & 0.00 & $0.00 \%$ \\
\hline & 28179587_Pla_MCI-AD2 & 8 & & 9 & $49.74 \%$ & & & & & & \\
\hline
\end{tabular}




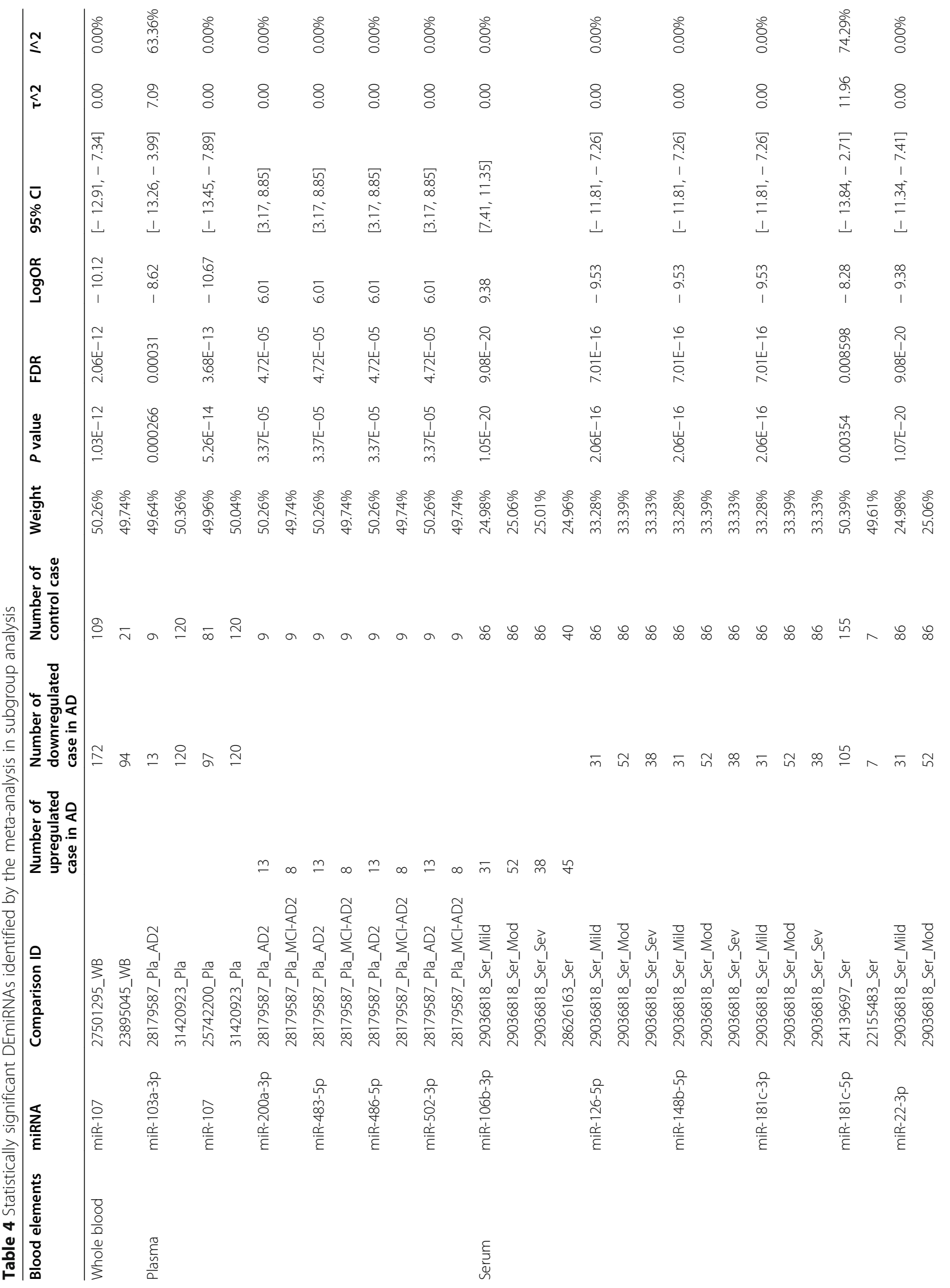




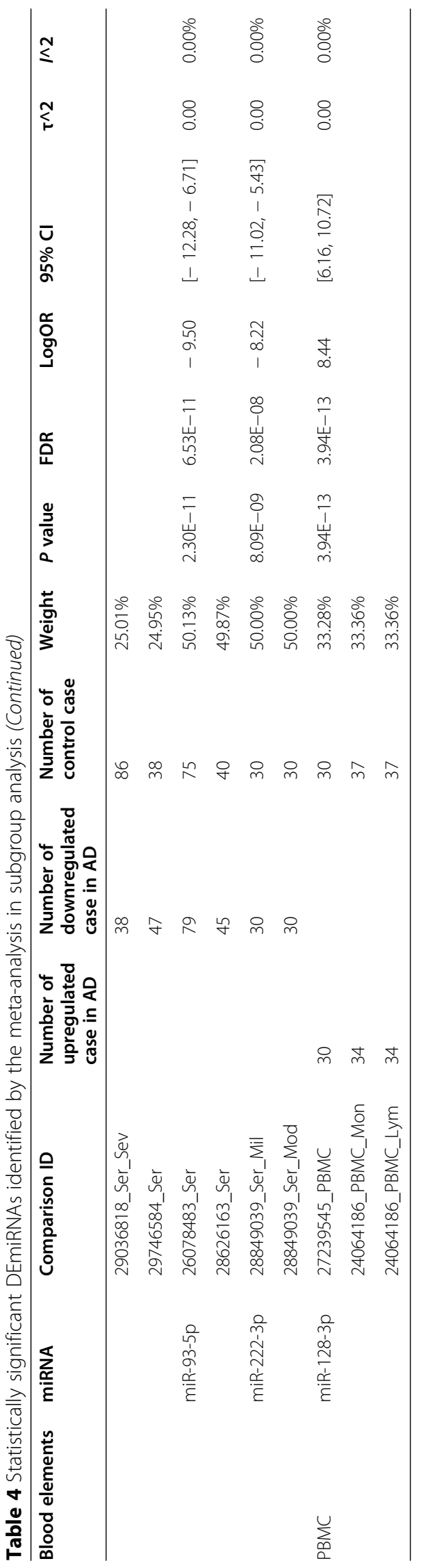


Table 5 AD-related DEmiRNAs identified by ABMDA

\begin{tabular}{lllll}
\hline Disease & miRNAs & Mature miRNAs & Score & Used as biomarkers in diseases \\
\hline Alzheimer disease & miR-339 & miR-339-5p & 9.45 & Lung cancer \\
& miR-128-2 & & 8.92 & Hepatocellular carcinoma \\
miR-203 & miR-203a-3p & 8.76 & Non-small cell lung cancer \\
miR-495 & miR-495-3p & 8.75 & AD \\
miR-155 & miR-155-5p & 8.70 & Lung cancer \\
let-7a-2 & & 8.67 & Breast cancer \\
miR-103a-2 & & 8.10 & Non-small cell lung cancer \\
miR-16-2 & & 8.02 & Malignant pleural mesothelioma \\
let-7b & let-7b-5p & 7.93 & 7.88 & miR-625-5p \\
miR-625
\end{tabular}

physiological function of the UPS can be adversely influenced by neuroinflammation. Under neuroinflammation, $\mathrm{J} 2$ prostaglandins are generated from prostaglandin D2, which is the most abundant prostaglandin in the brain [142]. J2 prostaglandins enhance the expression levels of COX2 to transition acute neuroinflammation to chronic neuroinflammation and oxidize the UPS units to promote disassembly [143]. The impaired UPS induces ectopic expression of cell cycle-related genes and causes neuronal CCR, as the metabolisms of cyclin and cyclin-dependent kinases are dependent on the UPS. A recent study [144] has reported that the dysregulation of an E3 ubiquitin ligase, Itch, induces neuronal CCR in response to $A \beta$. $A \beta$ induced JNK activation phosphorylates Itch to promote the degradation of TAp73, which is important for protein synthesis under oxidative stress, in neurons [145].

\section{Quality assessment of studies}

The MIQE guideline was used to access the expression profiling analysis of the included studies. The results of the quality assessments were shown in Fig. 4. Among the 47 expression profiling studies, miR-39 and U6 RNAs were frequently used as internal normalization controls for qRT-PCR. Around 85\% of the included studies provided sufficient information about data processing, including statistical analysis and quantification methods. About $70 \%$ of the included studies provided sufficient information about sample annotation, but 16 studies did not provide details of the storage or extraction methods of serum or plasma. Approximately $60 \%$ of the included studies did not provide the number of replicates in the experimental design, and 77\% did not provide the quantification cycle value in the actual data processing. The parameters of qRT-PCR methods were missing in 14 studies. For the annotation of PCR, most studies provided the full details of reference miRNAs for quantification, but primer information was missing in 19 studies.

\section{Discussion}

There is a need for blood circulating biomarkers that can be mass screened accurately and conveniently to identify high risk individuals of $\mathrm{AD}$. The identification of DEmiRNAs as biomarkers from differential miRNA expression studies has been successful in cancers [146] and is thought to have potential for $\mathrm{AD}$. DEmiRNAs associated with $\mathrm{AD}$ pathology, such as $A \beta$ production and neuroinflammation, are potentially important biomarkers because the presence of $A \beta$ and proinflammatory cytokines are considered to be key factors for predicting whether patients with mild cognitive impairment are progressing to $\mathrm{AD}$ [147-149]. In this study, we used a meta-analysis approach based on differential miRNA expression studies from blood to identify reliable miRNA$\mathrm{AD}$ associations. The associations were subsequently used for the prediction of potential $\mathrm{AD}$ biomarkers using the ABMDA ensemble learning method. We identified 28 DEmiRNAs (18 and 10 from meta-analysis and ABMDA, respectively) as potential $\mathrm{AD}$ biomarkers in blood.

The DEmiRNAs identified with the meta-analysis involved in $\mathrm{A} \beta$ metabolism, including $A P P$ expression, $\mathrm{A} \beta$ production enzyme regulation, and $A \beta$ clearance, tau protein phosphorylation, and also contribute to neuronal function during $\mathrm{AD}$ progression, including pathogenic neuroinflammation, apoptosis, mitochondrial oxygen chain activity, and neuronal microtubule maintenance. The meta-analytical results of DEmiRNAs mediate $A \beta$ synthesis via several targets, and some of them are also involved in tau protein phosphorylation and neuronal functions. MiR-107 is negatively correlated with $B A C E 1$ and $A D A M 10$ expression and is downregulated in the early stage of $\mathrm{AD}[150,151]$. The downregulation of miR-107 also dysregulates the expression of CDK5R1, which is involved in neuronal survival [94]. Downregulation of miR-181c dysregulates the expression of SPTLC1 [152], to increase $A \beta$ deposition, and increases proinflammatory cytokines [153]. MiR-22-3p and miR-29c also regulate $A \beta$ deposition via targeting MAPK14 and $B A C E 1$, respectively $[154,155]$. Besides $A \beta$ synthesis, $A \beta$ 


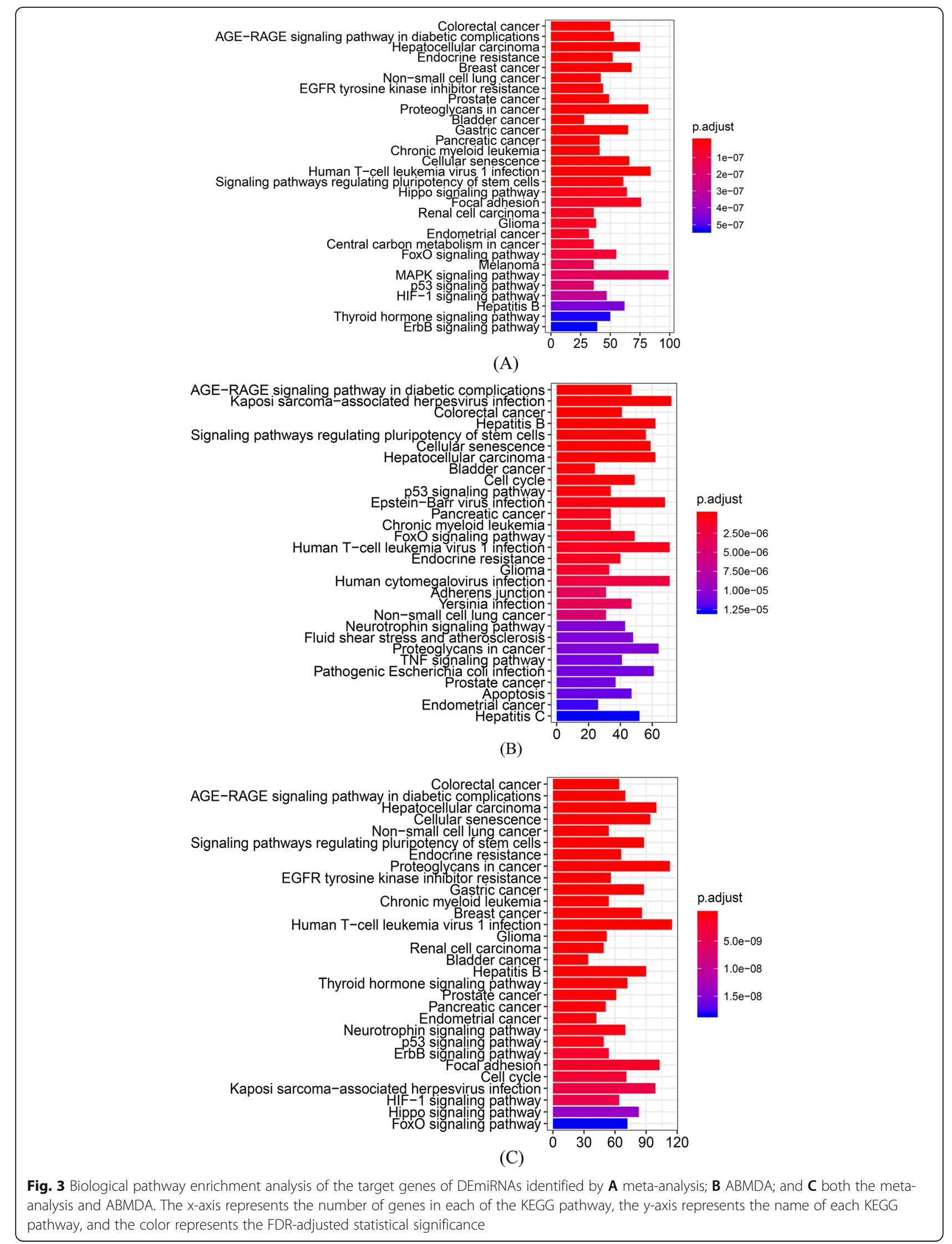




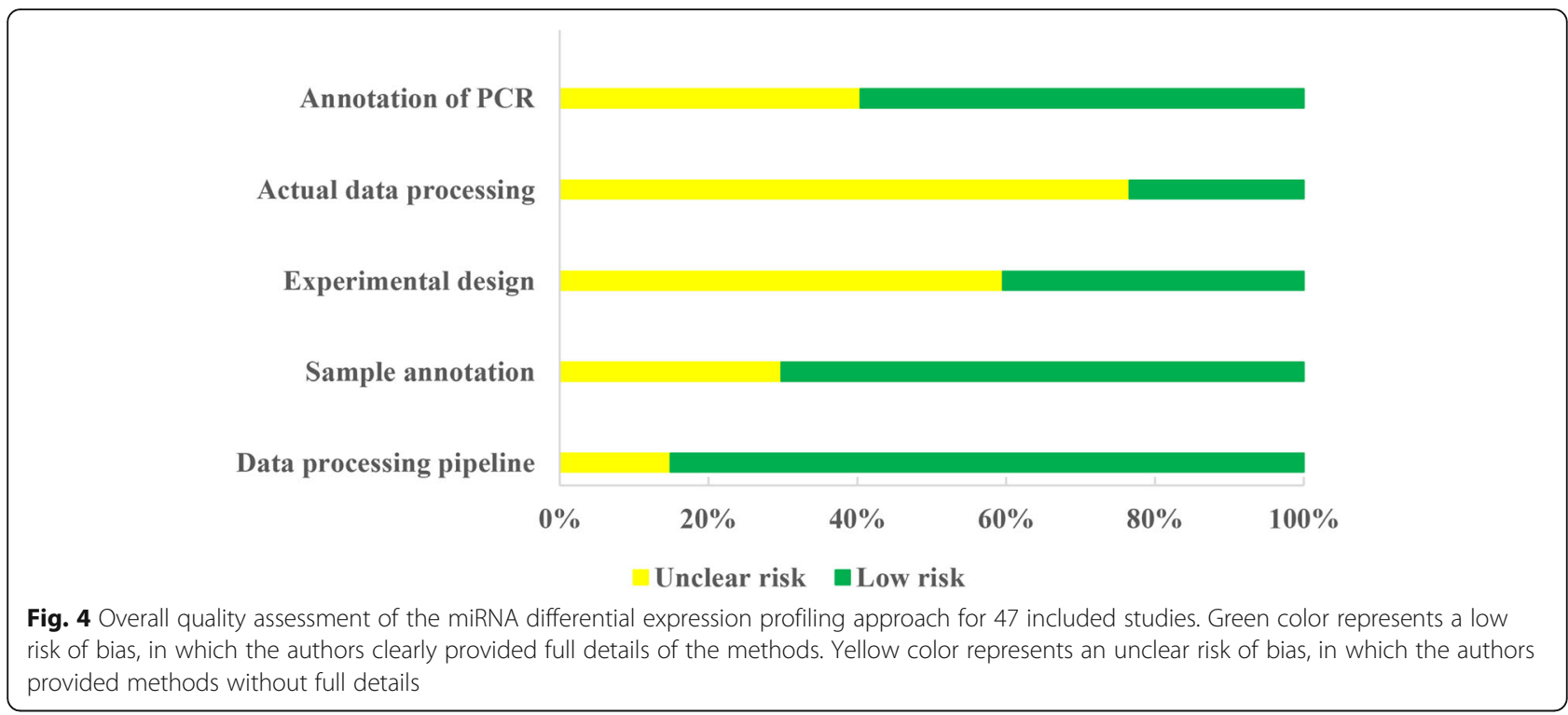

clearance is also interfered by the meta-analytical results of DEmiRNAs. MiR-128 and miR-93 are reported to be involved in $A \beta$ phagocytosis and UPS for $A \beta$ clearance by targeting cathepsin and NEDD4L, respectively $[87,156]$. For $A \beta$-induced toxicity, upregulation of miR-34c enhances $A \beta$-induced synaptic failure to suppress the memory formation by targeting SIRT1 and VAMP2 [157, 158]. MiR-200a-3p coregulates BACE1 and PRKACB to protect neurons against $A \beta$-induced toxicity and tau protein hyperphosphorylation [159], while upregulation of miR200a-3p also interferes the function of mitochondrial oxidative chain [69]. The meta-analytical results of DEmiRNAs were also involved in the tau protein phosphorylation. MiR-132 is consistently downregulated in different brain area, and negatively correlated with Braak stage, suggesting that it has an important role in cognitive capacity and correlated with tau protein phosphorylation $[160,161]$. The downregulation of miR-132 is reported to increase the expression of ITPKB and SIRT1 for tau pathology and $A \beta$ generation, respectively [162, 163]. Meanwhile, miR-132 is also involved in caspase 3dependent apoptosis [164]. MiR-483-5p and miR-106b target MAPT and FYN for tau protein synthesis and phosphorylation, respectively $[69,165]$. For neuronal function, miR-222 targets CDKN1B to influence cell cycle and apoptosis [68]; miR-191 targets TMOD2 and REST to regulate axonal guidance and dendritic growth [166]; and both miR-486-5p and miR-502-3p are the regulators of dynactin for neuronal function [69]. The potential mechanisms of miR-126-5p, miR-148-5p, and let-7d in AD are not well known. MiR-126-5p targets JNK-interacting protein 2 to mediate inflammatory response in infectious conditions [167]. The expression of miR-148-5p is reported to be positively correlated with MMSE score [66], and let-7d is reported to be involved in neuronal cell cycle by regulating enzymatic signaling [168]. Compared with other let-7 family members, let-7d does not significantly trigger the release of TNF- $\alpha$ from microglia [169].

DEmiRNAs from ABMDA also relate to neuroinflammation mainly via modulation of NF- $\mathrm{kB}$. The DEmiRNAs identified in the present study are compatible with the first two differential miRNA expression studies in AD, which reported that the DEmiRNAs are associated with dysregulated inflammation [23, 170]. Independent enrichment analysis in the present study indicated disrupted signaling of AGE-RAGE, cellular senescence, Hippo, FoxO, and cell cycles, and these dysregulated pathways were more statistically significant in the synergic enrichment analysis. The dysregulated pathways were associated with neuroinflammation, in which neuroinflammation increases the production of $A \beta$ by modulation of secretase, suggesting a biological significance of the 28 DEmiRNAs in AD development. The similarity of biological functions of the two DEmiRNA categories fits the ABMDA assumption that the miRNAs associated with the same disease should be functionally related. In the network, 1865 target genes were commonly found from the two DEmiRNA categories, and the highly connected target genes involved $U B C$, $U B B$, and RPS27A, which are involved in the neuroinflammatory process and CCR through mediating the UPS.

Comparison of DEmiRNA results from the metaanalysis and ABMDA revealed that the cell cycle pathway was identified as significant only with ABMDA. The majority of neuronal death in $\mathrm{AD}$ is due to the dysregulated cell cycle, in which the differentiated neurons in $\mathrm{AD}$-affected brain regions re-enter the cell cycle in the presymptomatic disease [171, 172]. The ectopic expression of developmentally regulated genes in $\mathrm{AD}$ links $\mathrm{AD}$ 
pathophysiology with aberrant CCR and correlates with cognitive decline [173]. CCR markers are expressed in $A \beta$-cultured neurons within hours, suggesting CCR is an initial event in response to $A \beta$ [174]. These findings indicate that neuronal $C C R$ induces rapid neuronal loss after exposure to $A \beta$. Neuronal CCR in AD results from $A \beta$-induced activation of multiple protein kinases at the plasma membrane, and tau protein phosphorylation by these proteins. The absence of $\beta$-secretases, or blockage of the $A \beta$ receptor, inhibits CCR $[175,176]$. $A \beta$ incorporates into the lipid rafts of neuronal membranes by Fyndependent kinase [177] and disturbs the structure of lipid rafts to activate PKA, CAMKII, Fyn, and mTORC1 to phosphorylate tau proteins at S409, S416, Y18, and S262, respectively $[126,127]$. The phosphorylated tau proteins subsequently modify mTORC1 activity to induce CCR. A $\beta$ also induces a rapid loss of the insulin receptor in the brain and impairs insulin receptor autophosphorylation to reduce brain insulin signaling [178], decreasing the inhibitory effects of MTORC1 at lysosomes in A $\beta$-induced CCR $[179,180]$. CCR can also be initiated by JNK, which is activated when $A \beta$ binds to receptors, such as RAGE, or by A $\beta$-induced TNF [144, 181]. Once CCR is initiated, neurons do not complete the cell cycle [171, 174]. The hyperploidy of neurons results in hypertrophy of neuronal cell bodies in ADaffected regions [182, 183]. The number of hyperploid neurons is higher in preclinical AD individuals than in healthy individuals, and initially increases followed by a gradual decrease in $\mathrm{AD}$ development [171]. The hyperploidy gradually decreases the synaptic inputs, which correlates with reduced activity of PSD-95 [129], a scaffold protein for glutamatergic function, suggesting that CCR-induced hyperploidy results in synaptic dysfunction. Further, loss of chromosomal homeostasis induces neuronal death [184], probably via neurotrophins and activation of FOXO1. Neurotrophins, such as nerve growth factor (NGF) and brain-derived neurotrophic factor (BDNF), elicit prosurvival functions, whereas their precursors, proNGF and proBDNF, elicit apoptotic functions. The levels of neurotrophins and their precursors are downregulated and upregulated in AD, respectively [185, 186], shifting neurotrophic prosurvival functions to apoptotic functions. Additionally, CCR induces dysregulation of cyclin metabolism to activate the transcription factor, FOXO1. The cyclin B-CDK1 complex, which is upregulated in $\mathrm{AD}$, phosphorylates FOXO1 [174] to induce the expression of apoptotic genes [187]. Thus, CCR is a key mechanism to functionally connect $A \beta$ and tau proteins in the early phase of $\mathrm{AD}$ development and connects the seemingly unrelated pathologies between $\mathrm{AD}$ and cancer, and between AD and insulin signaling impairment [188]. This convergence of pathology points to the importance of CCR in AD development and significance of miRNAs involved in CCR. Figure 5 summarizes the mechanism of $\mathrm{A} \beta$-mediated $\mathrm{CCR}$ in $\mathrm{AD}$, as described above.

Although it remains unclear whether neuroinflammation is the primary cause of $\mathrm{AD}$ development or a secondary event to other primary pathologies, neuroinflammation is an imperative and early event for $A \beta$ mediated toxicity and has been suggested to contribute as much as $A \beta$ in $A D$ pathology [189]. The level of neuroinflammation assessed by microglial activation has been correlated with worse cognitive decline in a PEG imaging study of AD participants [190]. Neuroinflammation is progressively increased during $\mathrm{AD}$ development, as indicated by elevated levels of proinflammatory cytokines [191]. Proinflammatory cytokines have been identified around amyloid plaques [192], suggesting a role of neuroinflammation in $A \beta$ deposition. Neuroinflammation impairs the microglial $A \beta$ degradation ability [193]. Several genome-wide association studies have reported that immune-related genes are involved in AD pathogenesis [194-197], such as CD33, CR1, EPHA1, and $M S 4 A 6 E / M S 4 A 4 E$, which regulate the immune system in response to $A \beta$ and activate microglial $A \beta$ degradation. An epigenome-wide association study [198] demonstrated hypermethylation of ANK1 in both presymptomatic and AD patients. ANK1 encodes Ankyrin 1 to maintain the actin cytoskeleton, and the upregulation of ANK1 in AD microglia has been reported, which supports the significance of the microglial response in AD development [199]. Passamonti et al. [200] reported that cognitive decline is mediated by microglial activation, which is linked to neuroinflammation. The perpetuation of inflammation influences synaptic plasticity and induces neuronal damage, resulting in neurodegeneration [201]. Synaptic loss results primarily from the increased levels of proinflammatory cytokines and activated microglial cells [202]. However, the importance of neuroinflammation in $\mathrm{AD}$ has been questioned because of the failure of nonsteroidal anti-inflammatory drugs (NSAIDs) for treating AD. An early AD clinical trial reported the failure of both cyclooxygenase-nonselective (naproxen) and cyclooxygenase-selective (celecoxib) NSAIDs in cognitive function improvement [203]. Another study [204], which involved long-term follow-up of $\mathrm{AD}$ participants who received naproxen or celecoxib, reported that naproxen reduces $\mathrm{AD}$ incidence in asymptomatic individuals. The trials suggest that the efficacy of NSAIDs is disease stage dependent and that the choice of NSAID is a determining factor. A COX nonselective NSAID, tarenflurbil, has shown some positive results at a high dosage for individuals with mild symptoms in a post hoc analysis of a phase II clinical trial [205], but no cognitive improvement was observed with the drug in a phase III clinical trial [206]. One possible reason for its failure is the low penetration of the drug 


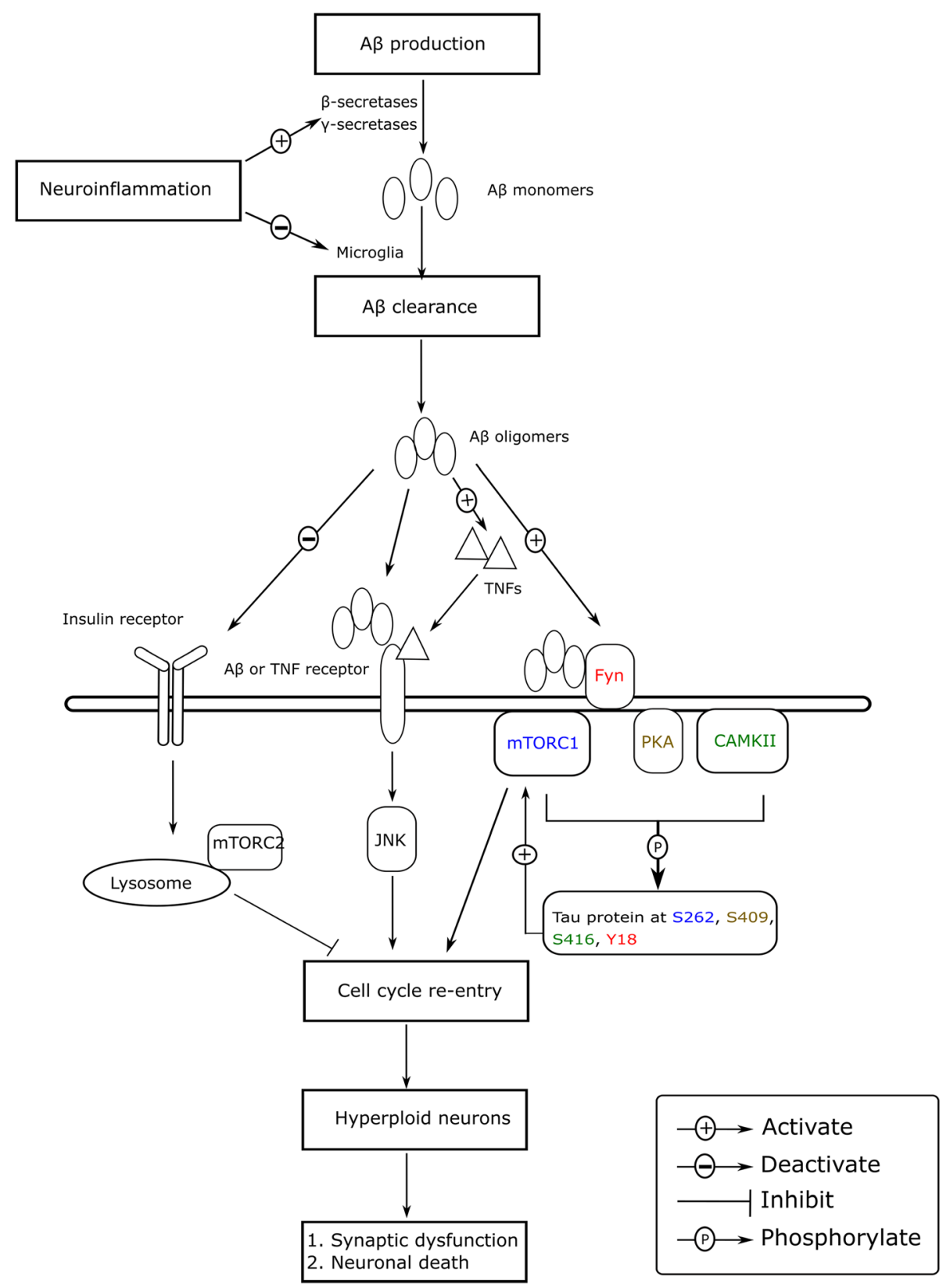

Fig. 5 Mechanism of action of neuronal $C C R$ in $A D$

from plasma to CSF. A recent study indicated that intranasal delivery of tarenflurbil can increase the drug concentration in the brain [207], but no clinical trials have yet been carried out with this route of delivery.

The miRNA expression profile in blood is representative of dysregulation in all tissues. The DEmiRNAs could be biased by the presence of multiple comorbidities, although the extent of how specific comorbidities influence DEmiRNAs in AD is not fully known. The miRNAs encapsulated in membrane vesicles can penetrate the BBB [208], suggesting that DEmiRNAs induced by peripheral complications could also contribute to $\mathrm{AD}$ pathology in the brain. Peripheral inflammation interferes with immunological processes in the brain through the entry of activated peripheral immune cells that exacerbate or initiate neuroinflammation. A growing body of evidence supports that peripheral inflammation is a driver of AD. For example, obesity and type 2 diabetes mellitus increase the risk of $\mathrm{AD}$ development through mediating neuroinflammation [209], and the gut microbial diversity in obesity patients is associated with increased levels of proinflammatory cytokines in blood 
[210]. The AD pathogenetic factor APOE \&4 allele has been reported to impair macrophage efferocytosis, which subsequently induces tissue inflammation and increases the circulating levels of proinflammatory cytokines [211, 212]. Elevated levels of proinflammatory cytokines in the blood of $\mathrm{AD}$ patients have been reported in metaanalyses [213, 214], confirming the presence of peripheral inflammation in AD. Peripheral inflammation is positively associated with cognitive decline and could detrimentally impact brain function [215]; specifically, elevated systemic TNF levels have been associated with increased conversion of mild cognitive impairment to AD. Systemic TNFs bind to TNF receptors to alter BBB integrity and allow entry of peripheral immune cells to the brain, inducing neuroinflammation [216, 217]. The entry of peripheral immune cells disrupts synaptic plasticity to induce neuroinflammation, increasing the risk of cognitive decline $[215,218]$. Neuroinflammation also releases antigens to activate $T$ cells in CSF. The activated $\mathrm{T}$ cells enter the brain and differentiate into effector $\mathrm{T}$ cells, which can produce cytokines [219] or induce apoptosis [220]. Abnormalities in $\mathrm{T}$ cells have been reported in AD patients compared with controls [221].

\section{Limitations}

Current approaches to diagnose AD (e.g., FDG-PET and CSF-based $A \beta$ and tau protein levels) require sophisticated equipment or lumbar puncture, which could be avoided with the use of blood-based biomarkers. The strength of this study is the integration of meta-analysis and the ABMDA ensemble learning method to identify potential AD biomarkers in blood. However, there are some limitations. First, most of the included miRNA expression studies only conducted qRT-PCR as a validation approach to identify DEmiRNAs, and only 17 of the included studies reported both screening and validation approaches. This means that the present identification of DEmiRNAs was dependent on those selected by researchers in previous studies, and not an objective manner. This implies that the number of DEmiRNAs in $\mathrm{AD}$ is likely larger than the number of DEmiRNAs identified in the present study. Second, although most DEmiRNAs from the meta-analysis and ABMDA identification were associated with neuroinflammation, their function was not distinguishable between acute and chronic neuroinflammation. Acute neuroinflammation is beneficial for microglial clearance of $A \beta$, whereas chronic neuroinflammation results in neurodegeneration by mediating functional and structural damage to neurons [222, 223]. Third, there were 9 DEmiRNAs from the meta-analysis extracted from the independent comparisons of only one study, suggesting that the meta-analytical results might be biased by the unidentified correlation within the recruited population.
Forth, disease stages of $\mathrm{AD}$ patients were not fully and consistently provided in most included differential miRNA expression studies, resulting in the performance of subgroup analysis based on disease stage was not currently applicable. Also, the results of original studies might be influenced by the lack of adjustment for the disease stages. Fifth, all the included differential miRNA expression studies were non-longitudinal studies, suggesting that the miRNAs extracted from these studies were not repeated observations over periods of time.

\section{Conclusion}

This study identified 28 DEmiRNAs as potential AD biomarkers in blood by meta-analysis and ABMDA ensemble learning in tandem. The DEmiRNAs identified by both meta-analysis and ABMDA were related to neuroinflammation, and those identified solely by ABMDA were related to neuronal CCR.

\section{Abbreviations \\ A $\beta$ : Amyloid-beta; ABMDA: Adaptive boosting for miRNA disease association; AD: Alzheimer's disease; AGE: Advanced glycation end product; BBB: Blood- brain barrier; BDNF: Brain-derived neurotrophins; CCR: Cell cycle re-entry; Cl: Confidence interval; CSF: Cerebrospinal fluid; DEmiRNAs: Differentially expressed microRNAs; FDG-PET: Fluorodeoxyglucose-positron emission tomography; FDR: False discovery rate; KEGG: Kyoto Encyclopedia of Genes and Genomes; logORs: Log odds ratios; miRNAs: MicroRNAs; MIQE: Minimum Information for Publication of Quantitative Real-time PCR Experiments; MMSE: Mini-Mental State Examination; NGF: Nerve growth factor; NIA- AA: National Institute of Aging and Alzheimer's Association; NINCDS- ADRDA: National Institute of Neurological and Communicative Disorder and Stroke and the Alzheimer's Disease and Related Disorders; NSAI \\ Ds: Nonsteroidal anti-inflammatory drugs; PBMC: Peripheral blood mononuclear cell; RAGE: Receptor for AGE; qRT-PCR: Quantitative real-time polymerase chain reaction; TNFs: Tumor necrosis factors; UPS: Ubiquitin- proteasome system; $\tau^{2}$ : Tau square}

\section{Supplementary Information}

The online version contains supplementary material available at https://doi. org/10.1186/s13195-021-00862-z.

Additional file 1. The meta-analysis results of DEmiRNAs.

Additional file 2. The ABMDA results.

Additional file 3. The target genes of the DEmiRNAs identified by meta-analysis and ABMDA.

\section{Acknowledgements}

We thank Lesley McCollum, PhD, from Edanz Group (https://en-authorservices.edanzgroup.com/ac) for editing a draft of this manuscript.

\section{Authors' contributions}

SWL and SCY conceived and designed the study. SCY, XNL, HMZ, and YLJ extracted the data. SCY and XNL analyzed the data. SCY, XNL, and SWL wrote the manuscript. $H M Z, Y L J$, and SWL verified the data and revised the manuscript. SCY and XNL contributed equally to the manuscript. All authors read and approved the final manuscript.

\section{Funding}

Part of the work of SWL was financially supported by the University of Edinburgh and the Shenzhen Institute of Artificial Intelligence and Robotics for Society (Grant No. 2021-ICP001). Part of this work was also supported by a grant (MYRG2019-00159-ICMS) received from the University of Macau. Part of the work of YLJ was financially supported by the Project of Basic Research 
Fund of Henan Institute of Medical and Pharmacological Sciences (2021BP0113) from Zhengzhou University.

\section{Availability of data and materials}

The raw data of this paper are available in the public database, PubMed. The data that support the findings of this paper are available in the published article and its additional files.

\section{Declarations}

\section{Ethics approval and consent to participate}

Not applicable. This study is a meta-analysis of published differential miRNA expression study.

\section{Consent for publication}

Not applicable.

\section{Competing interests}

The authors declare that they have no competing interests.

\section{Author details}

'State Key Laboratory of Quality Research in Chinese Medicine, Institute of Chinese Medical Sciences, University of Macau, Avenida da Universidade, Taipa 999078, Macao, China. ${ }^{2}$ BGI College \& Henan Institute of Medical and Pharmaceutical Sciences, Zhengzhou University, Zhengzhou, Henan, China. ${ }^{3}$ Department of Obstetrics and Gynecology, The Second Affiliated Hospital of Zhengzhou University, Zhengzhou, Henan, China. ${ }^{4}$ Shenzhen Institute of Artificial Intelligence and Robotics for Society, Shenzhen, China. ${ }^{5}$ Edinburgh Bayes Centre for Al Research in Shenzhen, College of Science and Engineering, University of Edinburgh, Edinburgh, Scotland, UK.

\section{Received: 17 October 2020 Accepted: 17 June 2021}

Published online: 09 July 2021

\section{References}

1. Guo T, Zhang D, Zeng Y, Huang TY, Xu H, Zhao Y. Molecular and cellular mechanisms underlying the pathogenesis of Alzheimer's disease. Mol Neurodegener. 2020;15:40 Available from: http://www.ncbi.nlm.nih.gov/ pubmed/32677986\%0A, http://www.pubmedcentral.nih.gov/articlerender. fcgi?artid=PMC7364557.

2. Zhao Y, Lukiw WJ. Bacteroidetes neurotoxins and inflammatory neurodegeneration. Mol Neurobiol. 2018;55:9100-7 Available from: http:// link.springer.com/10.1007/s12035-018-1015-y.

3. Andrade-Moraes $\mathrm{CH}$, Oliveira-Pinto AV, Castro-Fonseca E, daSilva CG, Guimaraes DM, Szczupak D, et al. Cell number changes in Alzheimer's disease relate to dementia, not to plaques and tangles. Brain. 2013;136: 3738-52 Available from: https://academic.oup.com/brain/article-lookup/ doi/10.1093/brain/awt273.

4. Thambisetty M, Lovestone S. Blood-based biomarkers of Alzheimer's disease: challenging but feasible. Biomark Med. 2010;4:65-79 Available from: https:// www.futuremedicine.com/doi/10.2217/bmm.09.84.

5. Dubois B, Feldman HH, Jacova C, deKosky ST, Barberger-Gateau P, Cummings J, et al. Research criteria for the diagnosis of Alzheimer's disease: revising the NINCDS-ADRDA criteria. Lancet Neurol. 2007:6:734-46 Available from: https://linkinghub.elsevier.com/retrieve/pii/S1474442207701783.

6. Jack CR, Bennett DA, Blennow K, Carrillo MC, Dunn B, Haeberlein SB, et al NIA-AA Research Framework: toward a biological definition of Alzheimer's disease. Alzheimers Dement. 2018;14:535-62 Available from: https:// linkinghub.elsevier.com/retrieve/pii/S1552526018300724.

7. Agrawal M, Biswas A. Molecular diagnostics of neurodegenerative disorders. Front Mol Biosci. 2015;2. Available from: https://www.frontiersin.org/a rticles/10.3389/fmolb.2015.00054/full. Accessed 22 Sept 2015.

8. McKhann GM, Knopman DS, Chertkow H, Hyman BT, Jack CR, Kawas CH, et al. The diagnosis of dementia due to Alzheimer's disease: recommendations from the National Institute on Aging-Alzheimer's Association workgroups on diagnostic guidelines for Alzheimer's disease. Alzheimers Dement. 2011;7:263-9 Available from: http://doi.wiley.com/10.1 016/j.jalz.2011.03.005.

9. Schindler SE, Fagan AM. Autosomal dominant Alzheimer disease: a unique resource to study CSF biomarker changes in preclinical AD. Front Neurol.
2015;6. Available from: https://www.frontiersin.org/articles/10.3389/fneur.201 5.00142/full. Accessed 29 June 2015.

10. Ritchie C, Smailagic N, Noel-Storr AH, Ukoumunne O, Ladds EC, Martin S. CSF tau and the CSF tau/ABeta ratio for the diagnosis of Alzheimer's disease dementia and other dementias in people with mild cognitive impairment (MCI). Cochrane Database Syst Rev. 2017;3:CD010803 Available from: http:// www.ncbi.nlm.nih.gov/pubmed/28328043.

11. Fei $M$, Jianghua W. RujuanM, Wei Z, QianW. The relationship of plasma $A \beta$ levels to dementia in aging individuals with mild cognitive impairment. J Neurol Sci. 2011;305:92-6 Available from: https://linkinghub.elsevier.com/ retrieve/pii/S0022510X11001146.

12. Koyama A, Okereke OI, Yang T, Blacker D, Selkoe DJ, Grodstein F. Plasma amyloid- $\beta$ as a predictor of dementia and cognitive decline: a systematic review and meta-analysis. Arch Neurol. 2012;69:824-31 Available from: http://archneur.jamanetwork.com/article.aspx?doi=10.1 001/archneurol.2011.1841.

13. Mattsson-Carlgren N, Janelidze S, Palmqvist S, Cullen N, Svenningsson AL, Strandberg $\mathrm{O}$, et al. Longitudinal plasma p-tau217 is increased in early stages of Alzheimer's disease. Brain. 2020;143:3234-41 Available from: http:// www.ncbi.nlm.nih.gov/pubmed/33068398.

14. Shin WS, Di J, Murray KA, Sun C, Li B, Bitan G, et al. Different amyloid- $\beta$ selfassemblies have distinct effects on intracellular tau aggregation. Front $\mathrm{Mol}$ Neurosci. 2019;12. Available from: https://www.frontiersin.org/article/10.33 89/fnmol.2019.00268/full. Accessed 8 Nov 2019.

15. Patel S, Shah RJ, Coleman P, Sabbagh M. Potential peripheral biomarkers for the diagnosis of Alzheimer's disease. Int J Alzheimers Dis. 2011;2011:1-9 Available from: http://www.hindawi.com/journals/ijad/2011/572495/.

16. O'Brien J, Hayder H, Zayed Y, Peng C. Overview of microRNA biogenesis, mechanisms of actions, and circulation. Front Endocrinol (Lausanne). 2018;9. Available from: https://www.frontiersin.org/article/10.3389/fendo.2018.004 02/full. Accessed 3 Aug 2018

17. Kozomara A, Birgaoanu M, Griffiths-Jones S. miRBase: from microRNA sequences to function. Nucleic Acids Res. 2019;47:D155-62 Available from: https://academic.oup.com/nar/article/47/D1/D155/5179337.

18. Brennan S, Keon M, Liu B, Su Z, Saksena NK. Panoramic visualization of circulating microRNAs across neurodegenerative diseases in humans. Mol Neurobiol. 2019;56:7380-407 Available from: http://link.springer.com/10.1 007/s12035-019-1615-1.

19. Hebert SS, Horre K, Nicolai L, Papadopoulou AS, Mandemakers W, Silahtaroglu AN, et al. Loss of microRNA cluster miR-29a/b-1 in sporadic Alzheimer's disease correlates with increased BACE1/ $\beta$-secretase expression. Proc Natl Acad Sci. 2008;105:6415-20 Available from: http://www.pnas.org/ cgi/doi/10.1073/pnas.0710263105.

20. Lei $X$, Lei L, Zhang Z, Zhang Z, Cheng Y. Downregulated miR-29c correlates with increased BACE1 expression in sporadic Alzheimer's disease. Int J Clin Exp Pathol. 2015;8:1565-74 Available from: http://www.ncbi.nlm.nih.gov/ pubmed/25973041.

21. Long JM, Ray B, Lahiri DK. MicroRNA-339-5p down-regulates protein expression of $\beta$-site amyloid precursor protein-cleaving enzyme 1 (BACE1) in human primary brain cultures and is reduced in brain tissue specimens of Alzheimer disease subjects. J Biol Chem. 2014;289:5184-98 Available from: http://www.jbc.org/lookup/doi/10.1074/jbc.M113.518241.

22. Wang $W-X$, Huang $Q, H u Y$, Stromberg AJ, Nelson PT. Patterns of microRNA expression in normal and early Alzheimer's disease human temporal cortex: white matter versus gray matter. Acta Neuropathol. 2011;121:193-205 Available from: http://link.springer.com/10.1007/s004 01-010-0756-0.

23. Schipper HM, Maes OC, Chertkow HM, Wang E. MicroRNA expression in Alzheimer blood mononuclear cells. Gene Regul Syst Bio. 2007;1:GRSB.S361 Available from: http://journals.sagepub.com/doi/10.4137/GRSB.S361.

24. Satoh J. Molecular network of microRNA targets in Alzheimer's disease brains. Exp Neurol. 2012;235:436-46 Available from: https://linkinghub. elsevier.com/retrieve/pii/S0014488611003104.

25. Cheng L, Quek CYJ, Sun X, Bellingham SA, Hill AF. The detection of microRNA associated with Alzheimer's disease in biological fluids using next-generation sequencing technologies. Front Genet. 2013;4. Available from: https://www.frontiersin.org/articles/10.3389/fgene.2013.00150/full. Accessed 8 Aug 2013.

26. Hu G, Drescher KM, Chen X-M. Exosomal miRNAs: biological properties and therapeutic potential. Front Genet. 2012;3. Available from: https://www. frontiersin.org/articles/10.3389/fgene.2012.00056/full. Accessed 20 Apr 2012. 
27. Kiko T, Nakagawa K, Tsuduki T, Furukawa K, Arai H, Miyazawa T. MicroRNAs in plasma and cerebrospinal fluid as potential markers for Alzheimer's disease. J Alzheimers Dis. 2014;39:253-9 Available from: https://www.medra. org/servlet/aliasResolver?alias=iospress\&doi=10.3233/JAD-130932.

28. Geekiyanage H, Jicha GA, Nelson PT, Chan C. Blood serum miRNA: Noninvasive biomarkers for Alzheimer's disease. Exp Neurol. 2012;235:491-6 Available from: https://inkinghub.elsevier.com/retrieve/pii/S001448861 1004304

29. Tan L, Yu J-T, Liu Q-Y, Tan M-S, Zhang W, Hu N, et al. Circulating miR-125b as a biomarker of Alzheimer's disease. J Neurol Sci. 2014;336:52-6 Available from: https://linkinghub.elsevier.com/retrieve/pii/S0022510X13029481.

30. Denk J, Oberhauser F, Kornhuber J, Wiltfang J, Fassbender K, Schroeter ML, et al. Specific serum and CSF microRNA profiles distinguish sporadic behavioural variant of frontotemporal dementia compared with Alzheimer patients and cognitively healthy controls. PLoS One. 2018;13:e0197329 Available from: https://dx.plos.org/10.1371/journal.pone.0197329. Yue J, editor.

31. Wu Y, Xu J, Xu J, Cheng J, Jiao D, Zhou C, et al. Lower serum levels of miR29c-3p and miR-19b-3p as biomarkers for Alzheimer's disease. Tohoku J Exp Med. 2017;242:129-36 Available from: https://www.jstage.jst.go.jp/article/ tjem/242/2/242_129/_article.

32. $\mathrm{Wu} H Z \mathrm{ZY}$, Ong KL, Seeher $\mathrm{K}$, Armstrong NJ, Thalamuthu A, Brodaty $H$, et al. Circulating microRNAs as biomarkers of Alzheimer's disease: a systematic review. J Alzheimers Dis. 2015;49:755-66 Available from: http://www.medra. org/servlet/aliasResolver?alias=iospress\&doi=10.3233/JAD-150619. Hornberger $M$, editor.

33. Zhao Y, Bhattacharjee S, Dua P, Alexandrov PN, Lukiw WJ. microRNA-based biomarkers and the diagnosis of Alzheimer's disease. Front Neurol. 2015;6. Available from: https://www.frontiersin.org/articles/10.3389/fneur.2015.001 62/full. Accessed 13 July 2015.

34. Takousis P, Sadlon A, Schulz J, Wohlers I, Dobricic V, Middleton L, et al. Differential expression of microRNAs in Alzheimer's disease brain, blood, and cerebrospinal fluid. Alzheimers Dement. 2019;15:1468-77 Available from: http://doi.wiley.com/10.1016/j.jalz.2019.06.4952

35. Hu Y-B, Li C-B, Song N, Zou Y, Chen S-D, Ren R-J, et al. Diagnostic value of microRNA for Alzheimer's disease: a systematic review and meta-analysis. Front Aging Neurosci. 2016;8. Available from: https://www.frontiersin.org/a rticles/10.3389/fnagi.2016.00013/full. Accessed 9 Feb 2016.

36. Zhang Y-H, Bai S-F, Yan J-Q. Blood circulating miRNAs as biomarkers of Alzheimer's disease: a systematic review and meta-analysis. Biomark Med. 2019;13:1045-54 Available from: https://www.futuremedicine.com/doi/1 0.2217/bmm-2018-0341.

37. Bloudek LM, Spackman DE, Blankenburg M, Sullivan SD. Review and metaanalysis of biomarkers and diagnostic imaging in Alzheimer's disease. J Alzheimers Dis. 2011;26:627-45 Available from: https://www.medra.org/ servlet/aliasResolver?alias=iospress\&doi=10.3233/JAD-2011-110458.

38. Lugli G, Cohen AM, Bennett DA, Shah RC, Fields CJ, Hernandez AG, et al. Plasma exosomal miRNAs in persons with and without Alzheimer disease: altered expression and prospects for biomarkers. PLoS One. 2015;10: e0139233 Available from: https://dx.plos.org/10.1371/journal.pone.0139233. Zhang B, editor.

39. Ludwig N, Fehlmann T, Kern F, Gogol M, Maetzler W, Deutscher S, et al. Machine learning to detect Alzheimer's disease from circulating non-coding RNAs. Genomics Proteomics Bioinformatics. 2019;17:430-40 Available from: https://linkinghub.elsevier.com/retrieve/pii/S1672022919301573.

40. Zhao X, Kang J, Svetnik V, Warden D, Wilcock G, David Smith A, et al. A machine learning approach to identify a circulating microRNA signature for Alzheimer disease. J Appl Lab Med. 2020;5:15-28 Available from: https://aca demic.oup.com/jalm/article/5/1/15/5690017.

41. Zhao $Y$, Chen $X$, Yin J. Adaptive boosting-based computational model for predicting potential miRNA-disease associations. Bioinformatics. 2019;35: 4730-8 Available from: https://academic.oup.com/bioinformatics/article/3 5/22/4730/5481952. Valencia A, editor.

42. Huang Z, Shi J, Gao Y, Cui C, Zhang S, Li J, et al. HMDD v3.0: a database for experimentally supported human microRNA-disease associations. Nucleic Acids Res. 2019;47:D1013-7 Available from: https://academic.oup.com/nar/a rticle/47/D1/D1013/5144956.

43. Moher D, Liberati A, Tetzlaff J, Altman DG. Preferred Reporting Items for Systematic Reviews and Meta-Analyses: the PRISMA Statement. J Clin Epidemiol. 2009;62:1006-12 Available from: https://linkinghub.elsevier.com/ retrieve/pii/S0895435609001796.

44. Haunsberger SJ, Connolly NMC, Prehn JHM. miRNAmeConverter: an R/ bioconductor package for translating mature miRNA names to different
miRBase versions. Bioinformatics. 2016;btw660. Available from: https://aca demic.oup.com/bioinformatics/article-lookup/doi/10.1093/bioinformatics/ btw660. Accessed 15 Feb 2017.

45. Viechtbauer W. Conducting meta-analyses in $\mathrm{R}$ with the metafor Package. J Stat Softw. 2010;36 Available from: http://www.jstatsoft.org/v36/i03/. Accessed 5 Aug 2010.

46. Yu G, Wang L-G, Yan G-R, He Q-Y. DOSE: an R/Bioconductor package for disease ontology semantic and enrichment analysis. Bioinformatics. 2015;31: 608-9 Available from: https://academic.oup.com/bioinformatics/articlelookup/doi/10.1093/bioinformatics/btu684.

47. Li J, Zhang S, Wan Y, Zhao Y, Shi J, Zhou Y, et al. MISIM v2.0: a web server for inferring microRNA functional similarity based on microRNA-disease associations. Nucleic Acids Res. 2019;47:W536-41 Available from: https:/aca demic.oup.com/nar/article/47/W1/W536/5487262.

48. Ru Y, Kechris KJ, Tabakoff B, Hoffman P, Radcliffe RA, Bowler R, et al. The multiMiR R package and database: integration of microRNA-target interactions along with their disease and drug associations. Nucleic Acids Res. 2014;42:e133 Available from: http://academic.oup.com/nar/article/42/1 7/e133/2902504/The-multiMiR-R-package-and-database-integration-of.

49. Huang HY, Lin YCD, Li J, Huang KY, Shrestha S, Hong HC, et al. miRTarBase 2020: updates to the experimentally validated microRNA-target interaction database. Nucleic Acids Res. 2019. Available from: https:/academic.oup.com/ nar/advance-article/doi/10.1093/nar/gkz896/5606625. Accessed 8 Jan 2020.

50. Yu G, Wang L-G, Han Y, He Q-Y. clusterProfiler: an R Package for comparing biological themes among gene clusters. Omi A J Integr Biol. 2012;16:284-7 Available from: http://www.liebertpub.com/doi/10.1089/omi.2011.0118.

51. Kanehisa M, Furumichi M, Tanabe M, Sato Y, Morishima K. KEGG: new perspectives on genomes, pathways, diseases and drugs. Nucleic Acids Res. 2017;45:D353-61 Available from: https://academic.oup.com/nar/articlelookup/doi/10.1093/nar/gkw1092.

52. Szklarczyk D, Gable AL, Lyon D, Junge A, Wyder S, Huerta-Cepas J, et al. STR NG v11: protein-protein association networks with increased coverage, supporting functional discovery in genome-wide experimental datasets. Nucleic Acids Res. 2019;47:D607-13 Available from: http://www.ncbi.nlm.nih. gov/pubmed/30476243.

53. Bustin SA, Benes V, Garson JA, Hellemans J, Huggett J, Kubista M, et al. The MIQE guidelines: minimum information for publication of quantitative realtime PCR experiments. Clin Chem. 2009;55:611-22 Available from: http:// www.ncbi.nlm.nih.gov/pubmed/19246619.

54. Kenny A, McArdle H, Calero M, Rabano A, Madden SF, Adamson K, et al. Elevated plasma microRNA-206 levels predict cognitive decline and progression to dementia from mild cognitive impairment. Biomolecules. 2019;9:734 Available from: https://www.mdpi.com/2218-273X/9/11/734.

55. Barbagallo C, Mostile G, Baglieri G, Giunta F, Luca A, Raciti L, et al. Specific signatures of serum miRNAs as potential biomarkers to discriminate clinically similar neurodegenerative and vascular-related diseases. Cell Mol Neurobiol. 2020;40:531-46 Available from: http://link.springer.com/10.1007/ s10571-019-00751-y.

56. Gámez-Valero A, Campdelacreu J, Vilas D, Ispierto L, Reñé R, Álvarez R, et al. Exploratory study on microRNA profiles from plasma-derived extracellular vesicles in Alzheimer's disease and dementia with Lewy bodies. Transl Neurodegener. 2019;8:31 Available from: https://translationalneurodegenera tion.biomedcentral.com/articles/10.1186/s40035-019-0169-5.

57. Yang Q, Zhao Q, Yin Y. miR-133b is a potential diagnostic biomarker for Alzheimer's disease and has a neuroprotective role. Exp Ther Med. 2019. Available from: http://www.spandidos-publications.com/10.3892/etm.2019. 7855. Accessed 5 Aug 2019.

58. Wang J, Chen C, Zhang Y. An investigation of microRNA-103 and microRNA107 as potential blood-based biomarkers for disease risk and progression of Alzheimer's disease. J Clin Lab Anal. 2020;34. Available from: https://onlinelibra ry.wiley.com/doi/abs/10.1002/jcla.23006. Accessed 16 Aug 2019.

59. Siedlecki-Wullich D, Català-Solsona J, Fábregas C, Hernández I, Clarimon J, Lleó $A$, et al. Altered microRNAs related to synaptic function as potential plasma biomarkers for Alzheimer's disease. Alzheimers Res Ther. 2019;11:46.

60. Zhou Q, Luo L, Wang X, Li X. Relationship between single nucleotide polymorphisms in the $3^{\prime} \mathrm{UTR}$ of amyloid precursor protein and risk of Alzheimer's disease and its mechanism. Biosci Rep. 2019;39. Available from: https://portlandpress.com/bioscirep/article/doi/10.1042/BSR20182485/21 9091/Relationship-between-single-nucleotide. Accessed 2 May 2019

61. Geng L, Zhang T, Liu W, Chen Y. Inhibition of miR-128 Abates A -mediated cytotoxicity by targeting PPAR- $\gamma$ via NF-KB inactivation in primary mouse 
cortical neurons and Neuro2a cells. Yonsei Med J. 2018:59:1096 Available from: https://eymj.org/DOlx.php?id=10.3349/ymj.2018.59.9.1096.

62. Dias IHK, Brown CL, Shabir K, Polidori MC, Griffiths HR. miRNA 933 expression by endothelial cells is increased by 27 -hydroxycholesterol and is more prevalent in plasma from dementia patients. J Alzheimers Dis. 2018; 64:1009-17 Available from: https://www.medra.org/servlet/aliasResolver?alia $s=$ iospress\&doi=10.3233/JAD-180201. Mecocci P, editor.

63. Wang Z, Qin W, Wei CB, Tang Y, Zhao LN, Jin HM, et al. The microRNA-1908 up-regulation in the peripheral blood cells impairs amyloid clearance by targeting ApoE. Int J Geriatr Psychiatry. 2018;33:980-6 Available from: http:// doi.wiley.com/10.1002/gps.4881.

64. Yang TT, Liu CG, Gao SC, Zhang Y, WangP C. The serum exosome derived microRNA-135a, -193b, and -384 were potential Alzheimer's disease biomarkers. Biomed Environ Sci. 2018;31:87-96 Available from: http://www. ncbi.n/m.nih.gov/pubmed/29606187.

65. Manzine PR, Pelucchi S, Horst MA, Vale FAC, Pavarini SCI, Audano M, et al. microRNA 221 targets ADAM10 mRNA and is downregulated in Alzheimer's disease. J Alzheimers Dis. 2017;61:113-23 Available from: https://www. medra.org/servlet/aliasResolver?alias=iospress\&doi=10.3233/JAD-170592.

66. Guo R, Fan G, Zhang J, Wu C, Du Y, Ye H, et al. A 9-microRNA signature in serum serves as a noninvasive biomarker in early diagnosis of Alzheimer's disease. J Alzheimers Dis. 2017;60:1365-77 Available from: https:/www. medra.org/servlet/aliasResolver?alias=iospress\&doi=10.3233/JAD-170343.

67. Kumar S, Vijayan M, Reddy PH. MicroRNA-455-3p as a potential peripheral biomarker for Alzheimer's disease. Hum Mol Genet. 2017;26:3808-22 Available from: https://academic.oup.com/hmg/article/26/19/3808/3976567.

68. Zeng Q, Zou L, Qian L, Zhou F, Nie H, Yu S, et al. Expression of microRNA-222 in serum of patients with Alzheimer's disease. Mol Med Rep. 2017;16:5575-9 Available from: https:/www.spandidos-publications.com/10.3892/mmr.2017.7301.

69. Nagaraj S, Laskowska-Kaszub K, Dębski KJ, Wojsiat J, Dąbrowski M, Gabryelewicz T, et al. Profile of 6 microRNA in blood plasma distinguish early stage Alzheimer's disease patients from non-demented subjects. Oncotarget. 2017;8:16122-43 Available from: https://www.oncotarget.com/ lookup/doi/10.18632/oncotarget.15109.

70. Hara N, Kikuchi M, Miyashita A, Hatsuta H, Saito Y, Kasuga K, et al. Serum microRNA miR-501-3p as a potential biomarker related to the progression of Alzheimer's disease. Acta Neuropathol Commun. 2017;5(1):10. https://doi. org/10.1186/s40478-017-0414-z.

71. Li W, Li X, Xin X, Kan P-C, Yan Y. MicroRNA-613 regulates the expression of brain-derived neurotrophic factor in Alzheimer's disease. Biosci Trends. 2016; 10:372-7 Available from: https:/www.jstage.jst.go.jp/article/bst/10/5/10_201 6.01127/_article.

72. Yılmaz ŞG, Erdal ME, Özge AA, Sungur MA. Can peripheral microRNA expression data serve as epigenomic (upstream) biomarkers of Alzheimer's disease? Omi A J Integr Biol. 2016;20:456-61 Available from: http://www. liebertpub.com/doi/10.1089/omi.2016.0099.

73. Zhang $Y$, Xing $H$, Guo S, Zheng Z, Wang H, Xu D. MicroRNA-135b has a neuroprotective role via targeting of $\beta$-site APP-cleaving enzyme 1. Exp Ther Med. 2016;12:809-14 Available from: https://www.spandidos-publica tions.com/10.3892/etm.2016.3366.

74. Xing H, Guo S, Zhang Y, Zheng Z, Wang H. Upregulation of microRNA-206 enhances lipopolysaccharide-induced inflammation and release of amyloid$\beta$ by targeting insulin-like growth factor 1 in microglia. Mol Med Rep. 2016; 14:1357-64 Available from: https://www.spandidos-publications.com/10.3 892/mmr.2016.5369.

75. Guedes JR, Santana I, Cunha C, Duro D, Almeida MR, Cardoso AM, et al. MicroRNA deregulation and chemotaxis and phagocytosis impairment in Alzheimer's disease. Alzheimers Dement Diagn Assess Dis Monit. 2016;3:7-17 Available from: https:/onlinelibrary.wiley.com/doi/abs/10.1016/j.dadm.2015.11.004.

76. Jia L-H, Liu Y-N. Downregulated serum miR-223 servers as biomarker in Alzheimer's disease. Cell Biochem Funct. 2016;34:233-7 Available from: http://doi.wiley.com/10.1002/cbf.3184

77. Ragusa M, Bosco P, Tamburello L, Barbagallo C, Condorelli AG, Tornitore M, et al. miRNAs plasma profiles in vascular dementia: biomolecular data and biomedical implications. Front Cell Neurosci. 2016;10. Available from: http:// journal.frontiersin.org/article/10.3389/fncel.2016.00051. Accessed 1 Mar 2016.

78. Ren R-J, Zhang Y-F, Dammer EB, Zhou Y, Wang L, Liu X-H, et al. Peripheral blood microRNA expression profiles in Alzheimer's disease: screening, validation, association with clinical phenotype and implications for molecular mechanism. Mol Neurobiol. 2016;53:5772-81 Available from: http://link.springer.com/10.1007/s12035-015-9484-8.
79. Dong H, Li J, Huang L, Chen X, Li D, Wang T, et al. Serum microRNA profiles serve as novel biomarkers for the diagnosis of Alzheimer's disease. Dis Markers. 2015;2015:1-11 Available from: http://www.hindawi.com/journals/ $\mathrm{dm} / 2015 / 625659 /$.

80. Yang G, Song Y, Zhou X, Deng Y, Liu T, Weng G, et al. MicroRNA-29c targets $\beta$ site amyloid precursor protein-cleaving enzyme 1 and has a neuroprotective role in vitro and in vivo. Mol Med Rep. 2015;12:3081-8 Available from: https:// www.spandidos-publications.com/10.3892/mmr.2015.3728.

81. Wang T, Chen K, Li H, Dong S, Su N, Liu Y, et al. The feasibility of utilizing plasma $<$ em $>$ MiRNA $107</$ em $>$ and $<$ em $>$ BACE $1</$ em $>$ messenger RNA gene expression for clinical diagnosis of amnestic mild cognitive impairment. J Clin Psychiatry. 2015;76:135-41 Available from: http://www. psychiatrist.com/jcp/article/pages/2015/v76n02/v76n0202.aspx.

82. Zhu Y, Li C, Sun A, Wang Y, Zhou S. Quantification of microRNA-210 in the cerebrospinal fluid and serum: implications for Alzheimer's disease. Exp Ther Med. 2015;9:1013-7 Available from: https://www.spandidos-publications. com/10.3892/etm.2015.2179.

83. Liu C, Wang J, Li L, Xue L, Zhang Y, Wang P. MicroRNA-135a and -200b, potential biomarkers for Alzheimer's disease, regulate $\beta$ secretase and amyloid precursor protein. Brain Res. 2014;1583:55-64 Available from: https://linkinghub.elsevier.com/retrieve/pii/S0006899314005435.

84. Liu C-G, Wang J-L, Li L, Wang P-C. MicroRNA-384 regulates both amyloid precursor protein and $\beta$-secretase expression and is a potential biomarker for Alzheimer's disease. Int J Mol Med. 2014;34:160-6 Available from: https:// www.spandidos-publications.com/10.3892/ijmm.2014.1780.

85. Tan L, Yu J-T, Tan M-S, Liu Q-Y, Wang H-F, Zhang W, et al. Genome-wide serum microRNA expression profiling identifies serum biomarkers for Alzheimer's disease. J Alzheimers Dis. 2014;40:1017-27 Available from: https:// www.medra.org/servlet/aliasResolver?alias=iospress\&doi=10.3233/JAD-132144.

86. Bhatnagar S, Chertkow H, Schipper HM, Yuan Z, Shetty V, Jenkins S, et al. Increased microRNA-34c abundance in Alzheimer's disease circulating blood plasma. Front Mol Neurosci. 2014;7. Available from: https://www.frontiersin. org/articles/10.3389/fnmol.2014.00002/full. Accessed 4 Feb 2014.

87. Tiribuzi R, Crispoltoni L, Porcellati S, DiLullo M, Florenzano F, Pirro M, et al. miR128 up-regulation correlates with impaired amyloid $\beta(1-42)$ degradation in monocytes from patients with sporadic Alzheimer's disease. Neurobiol Aging. 2014;35:345-56 Available from: https://linkinghub.elsevier.com/ retrieve/pii/S0197458013003370.

88. Kumar P, Dezso Z, Mackenzie C, Oestreicher J, Agoulnik S, Byrne M, et al. Circulating miRNA biomarkers for Alzheimer's disease. PLOS One. 2013;8:e69807 Available from: https://dx.plos.org/10.1371/journal.pone.0069807. Hill AF, editor.

89. Leidinger P, Backes C, Deutscher S, Schmitt K, Mueller SC, Frese K, et al. A blood based 12-miRNA signature of Alzheimer disease patients. Genome Biol. 2013;14:R78 BioMed Central Ltd.

90. Villa C, Ridolfi E, Fenoglio C, Ghezzi L, Vimercati R, Clerici F, et al. Expression of the transcription Factor Sp1 and its regulatory hsa-miR-29b in peripheral blood mononuclear cells from patients with Alzheimer's disease. J Alzheimers Dis. 2013;35:487-94 Available from: https://www.medra.org/ servlet/aliasResolver?alias=iospress\&doi=10.3233/JAD-122263.

91. Cao F, Liu Z, Sun G. Diagnostic value of miR-193a-3p in Alzheimer's disease and miR-193a-3p attenuates amyloid- $\beta$ induced neurotoxicity by targeting PTEN. Exp Gerontol. 2020;130:110814 Available from: https://inkinghub. elsevier.com/retrieve/pii/S0531556519305479.

92. Cha DJ, Mengel D, Mustapic M, Liu W, Selkoe DJ, Kapogiannis D, et al. MiR212 and miR-132 are downregulated in neurally derived plasma exosomes of Alzheimer's patients. Front Neurosci. 2019;13:1208.

93. Jiao Y, Kong L, Yao Y, Li S, Tao Z, Yan Y, et al. Osthole decreases beta amyloid levels through up-regulation of miR-107 in Alzheimer's disease. Neuropharmacology. 2016;108:332-44 Available from: https://inkinghub. elsevier.com/retrieve/pii/S0028390816301861.

94. Moncini S, Salvi A, Zuccotti P, Viero G, Quattrone A, Barlati S, et al. The role of miR-103 and miR-107 in regulation of CDK5R1 expression and in cellular migration. PLoS One. 2011;6:e20038 Available from: https://dx.plos.org/10.13 71/journal.pone.0020038. Polymenis M, editor.

95. Hébert SS, Horré K, Nicolaï L, Bergmans B, Papadopoulou AS, Delacourte A, et al. MicroRNA regulation of Alzheimer's amyloid precursor protein expression. Neurobiol Dis. 2009;33:422-8 Available from: https://inkinghub. elsevier.com/retrieve/pii/S0969996108002982.

96. Zhang Y, Wang J, Liu X, Li J, Fan S. MicroRNA miR-103a-3p targets NPAS3 to regulate progression of Alzheimer's disease. Trop J Pharm Res. 2020;19: 1015-21. 
97. Manzano-Crespo M, Atienza M, Cantero JL. Lower serum expression of miR-181c-5p is associated with increased plasma levels of amyloid-beta $1-40$ and cerebral vulnerability in normal aging. Transl Neurodegener. 2019;8:1-10.

98. Guo W-G, Zhang Y, Ge D, Zhang Y-X, Lu C-L, Wang Q, et al. Bioinformatics analyses combined microarray identify the desregulated microRNAs in lung cancer. Eur Rev Med Pharmacol Sci. 2013;17:1509-16 Available from: http:// www.ncbi.nlm.nih.gov/pubmed/23771539.

99. Zhuang $L, X u L$, Wang $P$, Meng Z. Serum miR-128-2 serves as a prognostic marker for patients with hepatocellular carcinoma. PLoS One. 2015;10: e0117274 Available from: https://dx.plos.org/10.1371/journal.pone.0117274 Navarro A, editor.

100. Chen X, Xu Y, Liao X, Liao R, Zhang L, Niu K, et al. Plasma miRNAs in predicting radiosensitivity in non-small cell lung cancer. Tumor Biol. 2016;37:11927-36 Available from: http://link.springer.com/10.1007/s132 77-016-5052-8.

101. Yanaihara N, Caplen N, Bowman E, Seike M, Kumamoto K, Yi M, et al. Unique microRNA molecular profiles in lung cancer diagnosis and prognosis. Cancer Cell. 2006;9:189-98 Available from: https://inkinghub. elsevier.com/retrieve/pii/S153561080600033X.

102. Shi W, Dong F, Jiang Y, Lu L, Wang C, Tan J, et al. Construction of prognostic microRNA signature for human invasive breast cancer by integrated analysis. Onco Targets Ther. 2019;12:1979-2010 Available from: https://www.dovepress.com/construction-of-prognostic-microrna-signaturefor-human-invasive-breas-peer-reviewed-article-OTT.

103. Zhou X, Zhang Z, Liang X. Regulatory network analysis to reveal important miRNAs and genes in non-small cell lung cancer. Cell J. 2020;21:459-66 Available from: http://www.ncbi.nlm.nih.gov/pubmed/31376328.

104. Kirschner MB, Cheng YY, Badrian B, Kao SC, Creaney J, Edelman JJB, et al. Increased circulating miR-625-3p: a potential biomarker for patients with malignant pleural mesothelioma. J Thorac Oncol. 2012;7:1184-91 Available from: https://linkinghub.elsevier.com/retrieve/pii/S1556086415332974.

105. Zhou Y, Xu Z, Yu Y, Cao J, Qiao Y, Qiao H, et al. Comprehensive analysis of the IncRNA-associated ceRNA network identifies neuroinflammation biomarkers for Alzheimer's disease. Mol Omi. 2019;15:459-69 Available from: http://xlink.rsc.org/?DOI=C9MO00129H.

106. Mei L, He M, Zhang C, Miao J, Wen Q, Liu X, et al. Paeonol attenuates inflammation by targeting HMGB1 through upregulating miR-339-5p. Sci Rep. 2019;9:19370 Available from: http://www.nature.com/articles/s41598-01 9-55980-4.

107. Andersson $\AA$, Covacu R, Sunnemark D, Danilov Al, Dal Bianco A, Khademi M, et al. Pivotal advance: HMGB1 expression in active lesions of human and experimental multiple sclerosis. J Leukoc Biol. 2008;84:1248-55 Available from: http://doi.wiley.com/10.1189/jlb.1207844

108. Zhang Y, Wei G, Di Z, Zhao Q. miR-339-5p inhibits alcohol-induced brain inflammation through regulating NF-kB pathway. Biochem Biophys Res Commun. 2014;452:450-6 Available from: https://linkinghub.elsevier.com/ retrieve/pii/S0006291X14015241.

109. Piñeiro-Hermida S, López IP, Alfaro-Arnedo E, Torrens R, Iñiguez M, AlvarezErviti $L$, et al. IGF1R deficiency attenuates acute inflammatory response in a bleomycin-induced lung injury mouse model. Sci Rep. 2017;7:4290 Available from: http://www.nature.com/articles/s41598-017-04561-4.

110. Xu H, Liu C, Zhang Y, Guo X, Liu Z, Luo Z, et al. Let-7b-5p regulates proliferation and apoptosis in multiple myeloma by targeting IGF1R. Acta Biochim Biophys Sin Shanghai. 2014;46:965-72 Available from: https://aca demic.oup.com/abbs/article-lookup/doi/10.1093/abbs/gmu089.

111. Jin H, Kim T-J, Choi J-H, Kim M-J, Cho Y-N, Nam K-I, et al. MicroRNA-155 as a proinflammatory regulator via SHIP-1 down-regulation in acute gouty arthritis. Arthritis Res Ther. 2014;16:R88 Available from: http://arthritis-resea rch.biomedcentral.com/articles/10.1186/ar4531.

112. Qian F-H, Deng X, Zhuang Q-X, Wei B, Zheng D-D. miR-625-5p suppresses inflammatory responses by targeting AKT2 in human bronchial epithelial cells. Mol Med Rep. 2019. Available from: http://www.spandidos-publica tions.com/10.3892/mmr.2019.9817. Accessed 3 Jan 2019.

113. Wang $X$, Jin $H$, Jiang $S$, Xu Y. MicroRNA-495 inhibits the high glucoseinduced inflammation, differentiation and extracellular matrix accumulation of cardiac fibroblasts through downregulation of NOD1. Cell Mol Biol Lett. 2018;23:23 Available from: https://cmbl.biomedcentral.com/articles/10.1186/ s1 1658-018-0089-X.

114. Lin X, Lin Q. MiRNA-495-3p attenuates TNF-a induced apoptosis and inflammation in human nucleus pulposus cells by targeting IL5RA.
Inflammation. 2020. Available from: http://link.springer.com/10.1007/s10753020-01254-5. Accessed 22 May 2020.

115. Cho KJ, Song J, Oh Y, Lee JE. MicroRNA-Let-7a regulates the function of microglia in inflammation. Mol Cell Neurosci. 2015;68:167-76 Available from: https://linkinghub.elsevier.com/retrieve/pii/S1044743115300051.

116. Caggiano R, Cattaneo F, Moltedo O, Esposito G, Perrino C, Trimarco B, et al. miR-128 is implicated in stress responses by targeting MAFG in skeletal muscle cells. Oxidative Med Cell Longev. 2017;2017:1-13 Available from: https://www.hindawi.com/journals/omcl/2017/9308310/.

117. Tian T, Zhou Y, Feng $X$, Ye S, Wang H, Wu W, et al. MicroRNA-16 is putatively involved in the NF-KB pathway regulation in ulcerative colitis through adenosine A2a receptor (A2aAR) mRNA targeting. Sci Rep. 2016;6: 30824 Available from: http://www.nature.com/articles/srep30824.

118. Lu Q, Ma Z, Ding Y, Bedarida T, Chen L, Xie Z, et al. Circulating miR-103a-3p contributes to angiotensin I-induced renal inflammation and fibrosis via a SNRK/NF-kB/p65 regulatory axis. Nat Commun. 2019;10:2145 Available from: http://www.nature.com/articles/s41467-019-10116-0.

119. Drenth H, Zuidema SU, Krijnen WP, Bautmans I, van der Schans C, Hobbelen $\mathrm{H}$. Advanced glycation end-products are associated with the presence and severity of paratonia in early stage Alzheimer disease. J Am Med Dir Assoc. 2017;18:636.e7-636.e12 Available from: https://linkinghub.elsevier.com/ retrieve/pii/S1525861017302190.

120. Miller MC, Tavares R, Johanson CE, Hovanesian V, Donahue JE, Gonzalez L, et al. Hippocampal RAGE immunoreactivity in early and advanced Alzheimer's disease. Brain Res. 2008;1230:273-80 Available from: https:// linkinghub.elsevier.com/retrieve/pii/S0006899308016053.

121. Wan W, Cao L, Liu L, Zhang C, Kalionis B, Tai X, et al. A 1-42 oligomerinduced leakage in an in vitro blood-brain barrier model is associated with up-regulation of RAGE and metalloproteinases, and down-regulation of tight junction scaffold proteins. J Neurochem. 2015;134:382-93 Available from: http://doi.wiley.com/10.1111/jnc.13122.

122. Tobon-Velasco J, Cuevas E, Torres-Ramos M. Receptor for AGEs (RAGE) as mediator of NF-kB pathway activation in neuroinflammation and oxidative stress. CNS Neurol Disord Drug Targets. 2014;13:1615-26.

123. Sastre M, Dewachter I, Rossner S, Bogdanovic N, Rosen E, Borghgraef $P$, et al. Nonsteroidal anti-inflammatory drugs repress $\beta$-secretase gene promoter activity by the activation of PPAR. Proc Natl Acad Sci. 2006; 103:443-8 Available from: http://www.pnas.org/cgi/doi/10.1073/pnas. 0503839103.

124. Yamamoto M, Kiyota T, Horiba M, Buescher JL, Walsh SM, Gendelman HE, et al. Interferon- $\gamma$ and tumor necrosis factor-a regulate amyloid- $\beta$ plaque deposition and $\beta$-secretase expression in Swedish mutant APP transgenic mice. Am J Pathol. 2007;170:680-92 Available from: https://linkinghub. elsevier.com/retrieve/pii/S0002944010608905.

125. Faden Al, Loane DJ. Chronic neurodegeneration after traumatic brain injury: Alzheimer disease, chronic traumatic encephalopathy, or persistent neuroinflammation? Neurotherapeutics. 2015;12:143-50 Available from: http://link.springer.com/10.1007/s13311-014-0319-5.

126. Norambuena A, Wallrabe H, McMahon L, Silva A, Swanson E, Khan SS, et al. mTOR and neuronal cell cycle reentry: how impaired brain insulin signaling promotes Alzheimer's disease. Alzheimers Dement. 2017;13:152-67 Available from: http://doi.wiley.com/10.1016/j.jalz.2016.08.015.

127. Seward ME, Swanson E, Norambuena A, Reimann A, Cochran JN, Li R, et al. Amyloid- $\beta$ signals through tau to drive ectopic neuronal cell cycle re-entry in Alzheimer's disease. J Cell Sci. 2013;126:1278-86 Available from: http://jcs. biologists.org/cgi/doi/10.1242/jcs.1125880.

128. Barrio-Alonso E, Fontana B, Valero M, Frade JM. Pathological aspects of neuronal hyperploidization in Alzheimer's disease evidenced by computer simulation. Front Genet. 2020;11. Available from: https://www.frontiersin. org/article/10.3389/fgene.2020.00287/full. Accessed 27 Mar 2020.

129. Barrio-Alonso E, Hernández-Vivanco A, Walton CC, Perea G, Frade JM. Cell cycle reentry triggers hyperploidization and synaptic dysfunction followed by delayed cell death in differentiated cortical neurons. Sci Rep. 2018;8: 14316 Available from: http://www.nature.com/articles/s41598-018-32708-4.

130. Bell KFS, Hardingham GE. The influence of synaptic activity on neuronal health. Curr Opin Neurobiol. 2011;21:299-305 Available from: https:// linkinghub.elsevier.com/retrieve/pii/S0959438811000067.

131. Musicco M, Adorni F, DiSanto S, Prinelli F, Pettenati C, Caltagirone C, et al. Inverse occurrence of cancer and Alzheimer disease: a population-based incidence study. Neurology. 2013;81:322-8 Available from: http://www. neurology.org/cgi/doi/10.1212/WNL.0b013e31829c5ec1. 
132. Boccardi V, Pelini L, Ercolani S, Ruggiero C, Mecocci P. From cellular senescence to Alzheimer's disease: the role of telomere shortening. Ageing Res Rev. 2015;22:1-8 Available from: https://linkinghub.elsevier.com/retrieve/ pii/S1568163715000379.

133. Flanary BE, Sammons NW, Nguyen C, Walker D, Streit WJ. Evidence that aging and amyloid promote microglial cell senescence. Rejuvenation Res. 2007;10:61-74 Available from: https://www.liebertpub.com/doi/10.1089/rej.2 006.9096.

134. Ribezzo F, Shiloh Y, Schumacher B. Systemic DNA damage responses in aging and diseases. Semin Cancer Biol. 2016;37-38:26-35 Available from: https://linkinghub.elsevier.com/retrieve/pii/S1044579X15300055.

135. Wei Z, Chen X-C, Song Y, Pan X-D, Dai X-M, Zhang J, et al. Amyloid $\beta$ protein aggravates neuronal senescence and cognitive deficits in 5XFAD mouse model of Alzheimer's disease. Chin Med J. 2016;129:1835-44 Available from: http://journals.lww.com/00029330-201608050-00013.

136. Ardestani A, Lupse B, Maedler K. Hippo signaling: key emerging pathway in cellular and whole-body metabolism. Trends Endocrinol Metab. 2018;29: 492-509 Available from: https://linkinghub.elsevier.com/retrieve/pii/S10432 76018300894.

137. Irwin M, Tare M, Singh A, Puli OR, Gogia N, Riccetti M, et al. A positive feedback loop of Hippo- and c-Jun-amino-terminal kinase signaling pathways regulates amyloid-beta-mediated neurodegeneration. Front Cell Dev Biol. 2020;8. Available from: https://www.frontiersin.org/article/10.3389/ fcell.2020.00117/full. Accessed 13 Mar 2020.

138. Cao D, Lu H, Lewis TL, Li L. Intake of sucrose-sweetened water induces insulin resistance and exacerbates memory deficits and amyloidosis in a transgenic mouse model of Alzheimer disease. J Biol Chem. 2007;282: 36275-82 Available from: http://www.jbc.org/lookup/doi/10.1074/jbc.M703 561200

139. Neumann K, Rojo L, Navarrete L, Farias G, Reyes P, Maccioni R. Insulin resistance and Alzheimers disease: molecular links \& clinical implications Curr Alzheimer Res. 2008;5:438-47 Available from: http://www.eurekaselect. com/openurl/content.php?genre=article\&issn=1567-2050\&volume=5\&issue $=$ $5 \&$ spage $=438$

140. Storz P. Forkhead homeobox type $O$ transcription factors in the responses to oxidative stress. Antioxid Redox Signal. 2011;14:593-605 Available from: http://www.liebertpub.com/doi/10.1089/ars.2010.3405.

141. Yuen SC, Zhu H, Leung S. A systematic bioinformatics workflow with metaanalytics identified potential pathogenic factors of Alzheimer's disease. Front Neurosci. 2020;14. Available from: https://www.frontiersin.org/article/1 0.3389/fnins.2020.00209/full. Accessed 3 Mar 2020

142. Ricciotti E, FitzGerald GA. Prostaglandins and inflammation. Arterioscler Thromb Vasc Biol. 2011;31:986-1000 Available from: https:/www.ahajourna Is.org/doi/10.1161/ATVBAHA.110.207449

143. Figueiredo-Pereira ME, Rockwell $P$, Schmidt-Glenewinkel T, Serrano $P$. Neuroinflammation and $J 2$ prostaglandins: linking impairment of the ubiquitin-proteasome pathway and mitochondria to neurodegeneration Front Mol Neurosci. 2015;7. Available from: https://www.frontiersin.org/a rticles/10.3389/fnmol.2014.00104/full. Accessed 13 Jan 2015.

144. Chauhan M, Modi PK, Sharma P. Aberrant activation of neuronal cell cycle caused by dysregulation of ubiquitin ligase Itch results in neurodegeneration. Cell Death Dis. 2020;11:441 Available from: http://www. nature.com/articles/s41419-020-2647-1.

145. Marini A, Rotblat B, Sbarrato T, Niklison-Chirou MV, Knight JRP, Dudek K, et al. TAp73 contributes to the oxidative stress response by regulating protein synthesis. Proc Natl Acad Sci. 2018;115:6219-24 Available from: http://www.pnas.org/lookup/doi/10.1073/pnas.1718531115.

146. Deyati A, Younesi E, Hofmann-Apitius M, Novac N. Challenges and opportunities for oncology biomarker discovery. Drug Discov Today. 2013; 18:614-24 Available from: https://linkinghub.elsevier.com/retrieve/pii/S13 59644612004370.

147. Morris JC, Roe CM, Grant EA, Head D, Storandt M, Goate AM, et al. Pittsburgh Compound B imaging and prediction of progression from cognitive normality to symptomatic Alzheimer disease. Arch Neurol. 2009;66 Available from: http://archneur.jamanetwork.com/article.aspx?doi=10.1001/a rchneurol.2009.269. Accessed Dec 2009.

148. Forsberg A, Engler $H$, Almkvist $O$, Blomquist $G$, Hagman $G$, Wall A, et al. PET imaging of amyloid deposition in patients with mild cognitive impairment. Neurobiol Aging. 2008;29:1456-65 Available from: https://linkinghub.elsevier. com/retrieve/pii/S019745800700139X
149. Furney SJ, Kronenberg D, Simmons A, Güntert A, Dobson RJ, Proitsi P, et al. Combinatorial markers of mild cognitive impairment conversion to Alzheimer's disease - cytokines and MRI measures together predict disease progression. J Alzheimers Dis. 2011;26:395-405 Available from: https://www. medra.org/servlet/aliasResolver?alias=iospress\&doi=10.3233/JAD-2011-0044. Ashford JW, Rosen A, Adamson M, Bayley P, SabriO, Furst A, et al. editors.

150. Wang W-X, Rajeev BW, Stromberg AJ, Ren N, Tang G, Huang Q, et al. The expression of microRNA miR-107 decreases early in Alzheimer's disease and may accelerate disease progression through regulation of beta-site amyloid precursor protein-cleaving enzyme 1. J Neurosci. 2008;28:1213-23 Available from: http://www.ncbi.nlm.nih.gov/pubmed/18234899.

151. Augustin R, Endres K, Reinhardt S, Kuhn P-H, Lichtenthaler SF, Hansen J, et al. Computational identification and experimental validation of microRNAs binding to the Alzheimer-related gene ADAM10. BMC Med Genet. 2012;13:35 Available from: http://www.ncbi.nlm.nih.gov/pubmed/22594617.

152. Geekiyanage $H$, Chan C. MicroRNA-137/181c regulates serine palmitoyltransferase and in turn amyloid $\beta$, novel targets in sporadic Alzheimer's disease. J Neurosci. 2011;31:14820-30 Available from: http:// www.ncbi.nlm.nih.gov/pubmed/21994399.

153. Hutchison ER, Kawamoto EM, Taub DD, Lal A, Abdelmohsen K, Zhang Y, et al. Evidence for miR-181 involvement in neuroinflammatory responses of astrocytes. Glia [Internet]. 2013;61:1018-28. Available from: http://doi.wiley. com/10.1002/glia.22483.

154. Ji Q, Wang X, Cai J, Du X, Sun H, Zhang N. MiR-22-3p regulates amyloid $\beta$ deposit in mice model of Alzheimer's disease by targeting mitogenactivated protein kinase 14. Curr Neurovasc Res. 2019;16:473-80 Available from: http://www.ncbi.nlm.nih.gov/pubmed/31713484.

155. Zong Y, Wang H, Dong W, Quan X, Zhu H, Xu Y, et al. miR-29c regulates BACE1 protein expression. Brain Res. 2011;1395:108-15 Available from: http://www.ncbi.n/m.nih.gov/pubmed/21565331.

156. Madadi S, Schwarzenbach $H$, Saidijam M, Mahjub R, Soleimani M. Potential microRNA-related targets in clearance pathways of amyloid- $\beta$ : novel therapeutic approach for the treatment of Alzheimer's disease. Cell Biosci. 2019:9:91 Available from: https://cellandbioscience.biomedcentral.com/a rticles/10.1186/s13578-019-0354-3.

157. Zovoilis A, Agbemenyah HY, Agis-Balboa RC, Stilling RM, Edbauer D, Rao P, et al. microRNA-34c is a novel target to treat dementias. EMBO J. 2011;30: 4299-308 Available from: http://www.ncbi.nlm.nih.gov/pubmed/21946562

158. Hu S, Wang H, Chen K, Cheng P, Gao S, Liu J, et al. MicroRNA-34C downregulation ameliorates amyloid- $\beta$-induced synaptic failure and memory deficits by targeting VAMP2. J Alzheimers Dis. 2015;48:673-86 Available from: https://www.medra.org/servlet/aliasResolver?alias= iospress\&doi=10.3233/JAD-150432.

159. Wang L, Liu J, Wang $Q$, Jiang H, Zeng L, Li Z, et al. MicroRNA-200a-3p mediates neuroprotection in Alzheimer-related deficits and attenuates amyloid-beta overproduction and tau hyperphosphorylation via coregulating BACE1 and PRKACB. Front Pharmacol. 2019;10:806 Available from: https://www.frontiersin.org/article/10.3389/fphar.2019.00806/full.

160. Hansen KF, Sakamoto K, Aten S, Snider KH, Loeser J, Hesse AM, et al. Targeted deletion of miR-132/-212 impairs memory and alters the hippocampal transcriptome. Learn Mem. 2016;23:61-71 Available from: http://www.ncbi.nlm.nih.gov/pubmed/26773099.

161. Smith PY, Hernandez-Rapp J, Jolivette F, Lecours C, Bisht K, Goupil C, et al. miR-132/212 deficiency impairs tau metabolism and promotes pathological aggregation in vivo. Hum Mol Genet. 2015;24:6721-35 Available from: http://www.ncbi.nlm.nih.gov/pubmed/26362250.

162. Salta E, Sierksma A, Vanden Eynden E, DeStrooper B. miR-132 loss derepresses ITPKB and aggravates amyloid and TAU pathology in Alzheimer's brain. EMBO Mol Med. 2016;8:1005-18 Available from: http://www.ncbi.nlm. nih.gov/pubmed/27485122.

163. Hernandez-Rapp J, Rainone S, Goupil C, Dorval V, Smith PY, Saint-Pierre M, et al. microRNA-132/212 deficiency enhances $A \beta$ production and senile plaque deposition in Alzheimer's disease triple transgenic mice. Sci Rep. 2016;6:30953 Available from: http://www.ncbi.nlm.nih.gov/pubmed/27484949.

164. El Fatimy R, Li S, Chen Z, Mushannen T, Gongala S, Wei Z, et al. MicroRNA132 provides neuroprotection for tauopathies via multiple signaling pathways. Acta Neuropathol. 2018;136:537-55 Available from: http://www. ncbi.nlm.nih.gov/pubmed/29982852.

165. Liu W, Zhao J, Lu G. miR-106b inhibits tau phosphorylation at Tyr18 by targeting Fyn in a model of Alzheimer's disease. Biochem Biophys Res 
Commun. 2016;478:852-7 Available from: http://www.ncbi.nlm.nih.gov/ pubmed/27520374.

166. Siedlecki-Wullich D, Miñano-Molina AJ, Rodríguez-Álvarez J. microRNAs as early biomarkers of Alzheimer's disease: a synaptic perspective. Cells. 2021; 10. Available from: http://www.ncbi.nlm.nih.gov/pubmed/33435363. Accessed Jan 2021.

167. Haidar M, Rchiad Z, Ansari HR, Ben-Rached F, Tajeri S, Latre De Late P, et al. miR-126-5p by direct targeting of JNK-interacting protein-2 (JIP-2) plays a key role in Theileria-infected macrophage virulence. PLoS Pathog. 2018;14: e1006942 Available from: http://www.ncbi.nlm.nih.gov/pubmed/29570727.

168. Fairchild CLA, Cheema SK, Wong J, Hino K, Simó S, La Torre A. Let-7 regulates cell cycle dynamics in the developing cerebral cortex and retina. Sci Rep. 2019;9:15336 Available from: http://www.ncbi.nlm.nih.gov/ pubmed/31653921.

169. Buonfiglioli A, Efe IE, Guneykaya D, Ivanov A, Huang Y, Orlowski E, et al. let-7 MicroRNAs regulate microglial function and suppress glioma growth through Toll-like receptor 7. Cell Rep. 2019;29:3460-3471.e7 Available from: http://www.ncbi.nlm.nih.gov/pubmed/31825829.

170. Cogswell JP, Ward J, Taylor IA, Waters M, Shi Y, Cannon B, et al. Identification of miRNA changes in Alzheimer's disease brain and CSF yields putative biomarkers and insights into disease pathways. J Alzheimers Dis. 2008;14:27-41 Available from: https://www.medra.org/servlet/aliasResolver?a lias=iospress\&doi=10.3233/JAD-2008-14103.

171. Arendt T, Brückner MK, Mosch B, Lösche A. Selective cell death of hyperploid neurons in Alzheimer's disease. Am J Pathol. 2010;177:15-20 Available from: https://linkinghub.elsevier.com/retrieve/pii/S000294401 0600594.

172. Herrup K, Yang Y. Cell cycle regulation in the postmitotic neuron: oxymoron or new biology? Nat Rev Neurosci. 2007;8:368-78 Available from: http:// www.nature.com/articles/nrn2124.

173. van Leeuwen LAG, Hoozemans JJM. Physiological and pathophysiological functions of cell cycle proteins in post-mitotic neurons: implications for Alzheimer's disease. Acta Neuropathol. 2015;129:511-25 Available from: http://link.springer.com/10.1007/s00401-015-1382-7.

174. Yang Y, Mufson EJ, Herrup K. Neuronal cell death is preceded by cell cycle events at all stages of Alzheimer's disease. J Neurosci. 2003;23:2557-63 Available from: http://www.jneurosci.org/lookup/doi/10.1523/JNEUROSCI.2307-02557.2003.

175. Varvel NH, Bhaskar K, Patil AR, Pimplikar SW, Herrup K, Lamb BT. A oligomers induce neuronal cell cycle events in Alzheimer's disease. J Neurosci. 2008;28: 10786-93 Available from: http:/www.jneurosci.org/cgi/doi/10.1523/ JNEUROSCI.2441-08.2008,

176. Kodis EJ, Choi S, Swanson E, Ferreira G, Bloom GS. N-methyl-D-aspartate receptor-mediated calcium influx connects amyloid- $\beta$ oligomers to ectopic neuronal cell cycle reentry in Alzheimer's disease. Alzheimers Dement. 2018; 14:1302-12 Available from: http://doi.wiley.com/10.1016/j.jalz.2018.05.017.

177. Williamson R, Usardi A, Hanger DP, Anderton BH. Membrane-bound $\beta$ amyloid oligomers are recruited into lipid rafts by a fyn-dependent mechanism. FASEB J. 2008;22:1552-9 Available from: https://onlinelibrary. wiley.com/doi/abs/10.1096/fj.07-9766com.

178. Zhao W, DeFelice FG, Fernandez S, Chen H, Lambert MP, Quon MJ, et al. Amyloid beta oligomers induce impairment of neuronal insulin receptors. FASEB J. 2008;22:246-60 Available from: https:/onlinelibrary.wiley.com/doi/a bs/10.1096/fj.06-7703com.

179. dela Monte SM. Type 3 diabetes is sporadic Alzheimer's disease: minireview. Eur Neuropsychopharmacol. 2014;24:1954-60 Available from: https:// linkinghub.elsevier.com/retrieve/pii/S0924977X1400176X.

180. Steen E, Terry BM, Rivera EJ, Cannon JL, Neely TR, Tavares R, et al. Impaired insulin and insulin-like growth factor expression and signaling mechanisms in Alzheimer's disease--is this type 3 diabetes? J Alzheimers Dis. 2005;7:63-80.

181. Bedse G, DiDomenico F, Serviddio G, Cassano T. Aberrant insulin signaling in Alzheimer's disease: current knowledge. Front Neurosci. 2015;9. Available from: https://www.frontiersin.org/articles/10.3389/fnins.2015.00204/full. Accessed 16 June 2015.

182. lacono D, O'Brien R, Resnick SM, Zonderman AB, Pletnikova O, Rudow G, et al. Neuronal hypertrophy in asymptomatic Alzheimer disease. J Neuropathol Exp Neurol. 2008;67:578-89 Available from: https://academic. oup.com/jnen/article-lookup/doi/10.1097/NEN.0b013e3181772794.

183. lacono D, Markesbery WR, Gross M, Pletnikova O, Rudow G, Zandi P, et al. The Nun study: clinically silent AD, neuronal hypertrophy, and linguistic skills in early life. Neurology. 2009;73:665-73 Available from: http://www. neurology.org/cgi/doi/10.1212/WNL.0b013e3181b01077.

184. Raina AK, Hochman A, Zhu X, Rottkamp CA, Nunomura A, Siedlak SL, et al. Abortive apoptosis in Alzheimer's disease. Acta Neuropathol. 2001;101:30510 Available from: http://link.springer.com/10.1007/s004010100378.

185. Ginsberg SD, Alldred MJ, Counts SE, Cataldo AM, Neve RL, Jiang Y, et al. Microarray analysis of hippocampal CA1 neurons implicates early endosomal dysfunction during Alzheimer's disease progression. Biol Psychiatry. 2010;68:885-93 Available from: https://linkinghub.elsevier.com/ retrieve/pii/S0006322310005299.

186. Peng S, Wuu J, Mufson EJ, Fahnestock M. Increased proNGF levels in subjects with mild cognitive impairment and mild Alzheimer disease. J Neuropathol Exp Neurol. 2004;63:641-9 Available from: https://academic. oup.com/jnen/article-lookup/doi/10.1093/jnen/63.6.641.

187. Yuan Z, Becker EBE, Merlo P, Yamada T, DiBacco S, Konishi Y, et al. Activation of FOXO1 by Cdk1 in cycling cells and postmitotic neurons. Science. 2008;319:1665-8 Available from: https:/www.sciencemag.org/ lookup/doi/10.1126/science.1152337.

188. Khan SS, Bloom GS. Tau: the center of a signaling nexus in Alzheimer's disease. Front Neurosci. 2016;10. Available from: https://www.frontiersin. org/articles/10.3389/fnins.2016.00031/full. Accessed 9 Feb 2016.

189. Zhang B, Gaiteri C, Bodea LG, Wang Z, McElwee J, Podtelezhnikov AA, et al. Integrated systems approach identifies genetic nodes and networks in lateonset Alzheimer's disease. Cell. 2013;153:707-20.

190. Edison P, Archer HA, Gerhard A, Hinz R, Pavese N, Turkheimer FE, et al. Microglia, amyloid, and cognition in Alzheimer's disease: An [11C](R)PK11195-PET and [11C]PIB-PET study. Neurobiol Dis. 2008;32:4129 Available from: https://linkinghub.elsevier.com/retrieve/pii/S09699961 08001885.

191. Belkhelfa M, Rafa H, Medjeber O, Arroul-Lammali A, Behairi N, Abada-Bendib $\mathrm{M}$, et al. IFN- $\gamma$ and TNF-a are involved during Alzheimer disease progression and correlate with nitric oxide production: a study in Algerian patients. J Interf Cytokine Res. 2014;34:839-47 Available from: http://www.liebertpub. com/doi/10.1089/ir.2013.0085.

192. Eikelenboom P, van Exel E, Hoozemans JJM, Veerhuis R, Rozemuller AJM. vanGoolWA. Neuroinflammation - an early event in both the history and pathogenesis of Alzheimer's disease. Neurodegener Dis. 2010;7:38-41 Available from: https://www.karger.com/Article/FullText/283480.

193. Thériault $P$, ElAli $A$, Rivest $S$. The dynamics of monocytes and microglia in Alzheimer's disease. Alzheimers Res Ther. 2015;7:41 Available from: http://a Izres.com/content/7/1/41.

194. Bertram L, Lange C, Mullin K, Parkinson M, Hsiao M, Hogan MF, et al. Genome-wide association analysis reveals putative Alzheimer's disease susceptibility loci in addition to APOE. Am J Hum Genet. 2008;83:623-32 Available from: https://linkinghub.elsevier.com/retrieve/pii/S000292 9708005430.

195. Lambert J-C, Heath S, Even G, Campion D, Sleegers K, Hiltunen M, et al. Genome-wide association study identifies variants at CLU and CR1 associated with Alzheimer's disease. Nat Genet. 2009:41:1094-9 Available from: http://www.nature.com/articles/ng.439.

196. Naj AC, Jun G, Beecham GW, Wang L-S, Vardarajan BN, Buros J, et al. Common variants at MS4A4/MS4A6E, CD2AP, CD33 and EPHA1 are associated with late-onset Alzheimer's disease. Nat Genet. 2011:43:436-41 Available from: http://www.nature.com/articles/ng.801.

197. Hollingworth P, Harold D, Sims R, Gerrish A, Lambert J-C, Carrasquillo MM, et al. Common variants at ABCA7, MS4A6A/MS4A4E, EPHA1, CD33 and CD2AP are associated with Alzheimer's disease. Nat Genet. 2011;43:429-35 Available from: http://www.nature.com/articles/ng.803.

198. Lunnon K, Smith R, Hannon E, DeJager PL, Srivastava G, Volta M, et al. Methylomic profiling implicates cortical deregulation of ANK1 in Alzheimer's disease. Nat Neurosci. 2014;17:1164-70 Available from: http://www.nature. com/articles/nn.3782.

199. Mastroeni D, Sekar S, Nolz J, Delvaux E, Lunnon K, Mill J, et al. ANK1 is upregulated in laser captured microglia in Alzheimer's brain; the importance of addressing cellular heterogeneity. PLoS One. 2017;12:e0177814 Available from: https://dx.plos.org/10.1371/journal.pone.0177814. Ginsberg SD, editor.

200. Passamonti L, Tsvetanov KA, Jones PS, Bevan-Jones WR, Arnold R, Borchert $\mathrm{RJ}$, et al. Neuroinflammation and functional connectivity in Alzheimer's disease: interactive influences on cognitive performance. J Neurosci. 2019; 39:7218-26 Available from: http://www.jneurosci.org/lookup/doi/10.1523/ JNEUROSCI.2574-18.2019. 
201. Yirmiya R, Goshen I. Immune modulation of learning, memory, neural plasticity and neurogenesis. Brain Behav Immun. 2011;25:181-213 Available from: https://linkinghub.elsevier.com/retrieve/pii/S0889159110005210.

202. Hong S, Beja-Glasser VF, Nfonoyim BM, Frouin A, Li S, Ramakrishnan S, et al. Complement and microglia mediate early synapse loss in Alzheimer mouse models. Science. 2016;352:712-6 Available from: https://www.sciencemag. org/lookup/doi/10.1126/science.aad8373.

203. Martin BK, Szekely C, Brandt J, Piantadosi S, Breitner JCS, Craft S, et al. Cognitive function over time in the Alzheimer's Disease Anti-inflammatory Prevention Trial (ADAPT). Arch Neurol. 2008;65:896 Available from: http://a rchneur.jamanetwork.com/article.aspx?doi=10.1001/archneur.2008.65.7. nct70006.

204. Breitner JC, Baker LD, Montine TJ, Meinert CL, Lyketsos CG, Ashe KH, et al. Extended results of the Alzheimer's disease anti-inflammatory prevention trial. Alzheimers Dement. 2011;7:402-11 Available from: http://doi.wiley. com/10.1016/j.jalz.2010.12.014.

205. Wilcock GK, Black SE, Hendrix SB, Zavitz KH, Swabb EA, Laughlin MA. Efficacy and safety of tarenflurbil in mild to moderate Alzheimer's disease: a randomised phase II trial. Lancet Neurol. 2008;7:483-93 Available from: https://linkinghub.elsevier.com/retrieve/pii/S1474442208700905.

206. Green RC, Schneider LS, Amato DA, Beelen AP, Wilcock G, Swabb EA, et al. Effect of tarenflurbil on cognitive decline and activities of daily living in patients with mild Alzheimer disease: a randomized controlled trial. JAMA. 2009;302:2557-64 Available from: http://jama.jamanetwork.com/article.a spx?doi=10.1001/jama.2009.1866

207. Muntimadugu E, Dhommati R, Jain A, Challa VGS, Shaheen M, Khan W. Intranasal delivery of nanoparticle encapsulated tarenflurbil: a potential brain targeting strategy for Alzheimer's disease. Eur J Pharm Sci. 2016;92: 224-34 Available from: https://linkinghub.elsevier.com/retrieve/pii/S092 8098716301701.

208. Blandford SN, Galloway DA, Moore CS. The roles of extracellular vesicle microRNAs in the central nervous system. Glia. 2018:2267-78. Available from: https://onlinelibrary.wiley.com/doi/abs/10.1002/glia.23445. Accessed 4 May 2018.

209. Takeda S, Sato N, Uchio-Yamada K, Sawada K, Kunieda T, Takeuchi D, et al. Diabetes-accelerated memory dysfunction via cerebrovascular inflammation and A deposition in an Alzheimer mouse model with diabetes. Proc Natl Acad Sci. 2010;107:7036-41 Available from: http://www.pnas.org/cgi/doi/1 0.1073/pnas.1000645107.

210. LeChatelier E, Nielsen T, Qin J, Prifti E, Hildebrand F, Falony G, et al. Richness of human gut microbiome correlates with metabolic markers. Nature. 2013; 500:541-6 Available from: http://www.nature.com/articles/nature12506.

211. Cash JG, Kuhel DG, Basford JE, Jaeschke A, Chatterjee TK, Weintraub NL, et al. Apolipoprotein E4 impairs macrophage efferocytosis and potentiates apoptosis by accelerating endoplasmic reticulum stress. J Biol Chem. 2012 287:27876-84 Available from: http://www.jbc.org/lookup/doi/10.1074/jbc. M112.377549.

212. Fond AM, Ravichandran KS. Clearance of dying cells by phagocytes: mechanisms and implications for disease pathogenesis. Adv Exp Med Biol. 2016:25-49 Available from: http://link.springer.com/10.1007/978-3-319-394 06-0_2. Accessed 25 Aug 2016.

213. Swardfager W, Lanctôt K, Rothenburg L, Wong A, Cappell J, Herrmann N. A meta-analysis of cytokines in Alzheimer's disease. Biol Psychiatry. 2010;68: 930-41 Available from: https://inkinghub.elsevier.com/retrieve/pii/ S0006322310006013

214. Lai KSP, Liu CS, Rau A, Lanctôt KL, Köhler CA, Pakosh M, et al. Peripheral inflammatory markers in Alzheimer's disease: a systematic review and metaanalysis of 175 studies. J Neurol Neurosurg Psychiatry. 2017:88:876-82 Available from: http://jnnp.bmj.com/lookup/doi/10.1136/jnnp-2017-316201.

215. Holmes C, Cunningham C, Zotova E, Woolford J, Dean C, Kerr S, et al. Systemic inflammation and disease progression in Alzheimer disease. Neurology [Internet]. 2009;73:768-74. Available from: http://www.neurology. org/cgi/doi/10.1212/WNL.0b013e3181b6bb95.

216. Sharma HS, Castellani RJ, Smith MA, Sharma A The blood-brain barrier in Alzheimer's disease: novel therapeutic targets and nanodrug delivery. Int Rev Neurobiol. 2012:47-90. Available from: https://inkinghub.elsevier.com/ retrieve/pii/B978012386986900003X. Accessed 27 June 2012.

217. Qin L, Wu X, Block ML, Liu Y, Breese GR, Hong J-S, et al. Systemic LPS causes chronic neuroinflammation and progressive neurodegeneration. Glia. 2007; 55:453-62 Available from: http://doi.wiley.com/10.1002/glia.20467.
218. Riazi K, Galic MA, Kuzmiski JB, Ho W, Sharkey KA, Pittman QJ. Microglial activation and TNFa production mediate altered CNS excitability following peripheral inflammation. Proc Natl Acad Sci. 2008;105:17151-6 Available from: http://www.pnas.org/cgi/doi/10.1073/pnas.0806682105.

219. Luckheeram RV, Zhou R, Verma AD, Xia B. CD4 + T cells: differentiation and functions. Clin Dev Immunol. 2012;2012:1-12 Available from: http://www. hindawi.com/journals/jir/2012/925135/.

220. Harty JT, Tvinnereim AR, White DW. CD8+ T cell effector mechanisms in resistance to infection. Annu Rev Immunol. 2000;18:275-308 Available from: http://www.annualreviews.org/doi/10.1146/annurev.immunol.18.1.275.

221. Fehervari Z. Lymphocytes in Alzheimer's disease. Nat Immunol. 2016;17:355 Available from: http://www.nature.com/articles/ni.3427.

222. Ji K, Akgul G, Wollmuth LP, Tsirka SE. Microglia actively regulate the number of functional synapses. PLoS One. 2013;8:e56293 Available from: https://dx. plos.org/10.1371/journal.pone.0056293. Dunaevsky A, editor.

223. Krabbe G, Halle A, Matyash V, Rinnenthal JL, Eom GD, Bernhardt U, et al. Functional impairment of microglia coincides with beta-amyloid deposition in mice with Alzheimer-like pathology. PLoS One. 2013;8:e60921 Available from: https://dx.plos.org/10.1371/journal.pone.0060921. Priller J, editor.

\section{Publisher's Note}

Springer Nature remains neutral with regard to jurisdictional claims in published maps and institutional affiliations.

\section{Ready to submit your research? Choose BMC and benefit from:}

- fast, convenient online submission

- thorough peer review by experienced researchers in your field

- rapid publication on acceptance

- support for research data, including large and complex data types

- gold Open Access which fosters wider collaboration and increased citations

- maximum visibility for your research: over $100 \mathrm{M}$ website views per year

At BMC, research is always in progress.

Learn more biomedcentral.com/submissions 